\title{
Loss-of-function mutations in TRAF7 and KLF4 cooperatively activate RAS-like GTPase signaling and promote meningioma development
}

Running title: Loss of TRAF7 and KLF4 promotes meningioma development Paul Najm ${ }^{1,2}$, Peihua Zhao ${ }^{1,2}$, Mikhail Steklov ${ }^{1,2}$, Raj Nayan Sewduth ${ }^{1,2}$, Maria Francesca Baietti ${ }^{1,2}$, Silvia Pandolfi ${ }^{1,2}$, Nathan Criem ${ }^{1,2}$, Benoit Lechat ${ }^{1,2}$, Teresa Mendes Maia ${ }^{3,4,5}$, Delphi Van Haver ${ }^{3,4,5}$, Nikky Corthout $^{6}$, Sven Eyckerman ${ }^{3,4}$, Francis Impens $^{3,4,5}$, Anna A Sablina ${ }^{1,2 *}$

${ }^{1}$ VIB-KU Leuven Center for Cancer Biology, VIB, 3000 Leuven, Belgium.

${ }^{2}$ Department of Oncology, KU Leuven, Herestraat 49, 3000 Leuven, Belgium.

${ }^{3}$ VIB Center for Medical Biotechnology, Technologiepark-Zwijnaarde 75, 9052 Ghent, Belgium.

${ }^{4}$ Department of Biomolecular Medicine, Ghent University, Technologiepark-Zwijnaarde 75, 9052 Ghent, Belgium.

${ }^{5}$ VIB Proteomics Core, Technologiepark-Zwijnaarde 75, 9052 Ghent, Belgium.

${ }^{6}$ VIB LiMoNe \& Leuven Bio Imaging Core, Herestraat 49, 3000 Leuven, Belgium.

* Corresponding Author: Anna A. Sablina, VIB-KU Leuven Center for Cancer Biology, Herestraat 49, Bus 912, Leuven, Belgium 3000.

Phone: +32 163769 27; E-mail: Anna.Sablina@cme.vib-kuleuven.be

Key Words: Meningioma, Ubiquitin System, small GTPases

\section{Acknowledgments}

The work was supported by H2020 European Research Council (ub-RASDisease, 772649).

The authors have declared that no conflict of interest exists. 


\begin{abstract}
Meningiomas are the most common benign brain tumors. Mutations of the E3 ubiquitin ligase TRAF7 occur in $25 \%$ of meningiomas and commonly co-occur with mutations in KLF4, yet the functional link between TRAF7 and KLF4 mutations remains unclear. By generating an in vitro meningioma model derived from primary meningeal cells, we elucidated the cooperative interactions that promote meningioma development. By integrating TRAF7-driven ubiquitinome and proteome alterations in meningeal cells and the TRAF7 interactome, we identified TRAF7 as a proteostatic regulator of RASrelated small GTPases. Meningioma-associated TRAF7 mutations disrupted either its catalytic activity or its interaction with RAS GTPases. TRAF7 loss in meningeal cells altered actin dynamics and promoted anchorage-independent growth by inducing CDC42 and RAS signaling. TRAF7 deficiencydriven activation of the RAS/MAPK pathway promoted KLF4-dependent transcription that led to upregulation of the tumor-suppressive Semaphorin pathway, a negative regulator of small GTPases. KLF4 loss-of-function disrupted this negative feedback loop and enhanced mutant TRAF7-mediated cell transformation. Overall, this study provides new mechanistic insights into meningioma development, which could lead to novel treatment strategies.
\end{abstract}

\title{
Significance
}

The intricate molecular crosstalk between the ubiquitin ligase TRAF7 and the transcription factor KLF4 provides a first step towards the identification of new therapies for meningioma patients. 


\section{Introduction}

Meningiomas are the most common benign intracranial tumors, accounting for about $35 \%$ of all primary brain tumors (1). Although surgery is the preferred treatment for the majority of meningioma patients, maximal surgical resection without compromising neurological functions can be challenging, and residual meningiomas following surgical resection have a high likelihood of recurrence. A comprehensive understanding of the functional basis underlying meningioma development may lead to more effective treatments of meningioma patients (2).

The most well-studied genomic alterations in meningiomas are monosomy of chromosome 22 and inactivating mutations of Neurofibromatosis 2 gene (NF2). Mutations in the TNF receptor-associated factor 7 gene TRAF7 are the second most frequent genetic alterations, found in about $25 \%$ of all grade I and grade II meningiomas (Figure 1A). TRAF7 mutations commonly co-occur with mutations in Kruppel-like factor 4 (KLF4), v-Akt murine thymoma viral oncogene homolog 1 (AKT1), or phosphatidylinositol-4,5-biphosphate 3-kinase catalytic subunit alpha $(P I K 3 C A)$ but are mutually exclusive with mutations in the Hedgehog pathway member Smoothened (SMO) and in NF2 (3).

TRAF7 is an E3 ubiquitin ligase containing an N-terminal RING finger domain and a C-terminal WD40 repeat domain responsible for the substrate recognition (Figure 1A). Even though TRAF7 was initially classified as a member of the tumor necrosis factor receptor-associated factor (TRAF) family, the lack of the classical TRAF domain suggests a misclassification (4). In addition to meningioma, TRAF7 mutations have been identified at a high frequency in adenomatoid tumors of the genital tract and intraneural perineuriomas, a rare nerve sheath tumor derived from perineurial cells $(5,6)$. TRAF7 mutations, mutually exclusive with $N F 2$ alterations, have also been identified in a subset of pleural mesotheliomas $(7,8)$. 
About $40 \%$ of TRAF7 mutated meningiomas harbor a recurrent KLF4-K409Q mutation, which occurs almost exclusively in meningiomas (Figure 1A). KLF4 belongs to a family of transcriptional regulators involved in proliferation, differentiation, migration, inflammation, and pluripotency (3). In cancer, KLF4 has been reported to act as both a tumor suppressor and an oncogene (9-11). A recent study demonstrated that decreased KLF4 expression in anaplastic meningioma stem-like cells promotes their tumorigenicity, suggesting a tumor suppressive role of KLF4 in anaplastic meningiomas (12). Nonetheless, it is still unclear how the $K 409 Q$ mutation alters the regulation of KLF4's target genes, eventually contributing to meningioma development $(13,14)$. Approximately a third of TRAF7 mutated meningiomas have $A K T 1$ mutations. The only recurrent oncogenic $A K T 1-E 17 K$ mutation is well-known to activate the PI3K/AKT pathway (1). The PI3K/AKT pathway could be also up-regulated in TRAF7mutated meningiomas due to mutations in PI3KCA (3).

The majority of TRAF7-mutated meningiomas are located at the skull base and are known to invade the neighboring brain parenchyma due to its intrusive growth and intimately associated with the surrounding tissues (2), challenging a complete surgical resection with no alternative treatment options. Understanding the mechanisms leading to non-NF2 mutated meningioma development may have a considerable translational impact by increasing therapeutic options for meningioma treatment. 


\section{Material and Methods}

\section{Cell culture and reagents}

HEK293T cells and CH157-MN cells (a kind gift of Dr. Ian Dunn, Brigham and Women's Hospital) were cultured in DMEM medium (GIBCO) supplemented with $10 \%$ fetal bovine serum and $1 \%$ penicillin/streptomycin. Meningioma HBL52 cells (Cell Lines Service) and primary Human Meningeal Cells (Sciencell Research Laboratories) were cultured according to the manufacturer's protocols. Cells were tested with a mycoplasma testing kit (Lonza MycoAlert, LT07-318) every two weeks.

Lentiviral infections were performed as described by the RNAi Consortium (TRC) (http://www.broadinstitute.org/rnai/public/resources/protocols). Infected cells were selected by treatment with $2 \mu \mathrm{g} / \mathrm{ml}$ puromycin (InvivoGen); $400 \mu \mathrm{g} / \mathrm{ml}$ of hygromycin-B (Thermo Fisher Scientific); or 500ug/ml G418 (InvivoGen) and then recovered for two-three weeks. Transient transfections were performed using GeneJuice (Millipore), Turbofect (ThermoScientific), or Lipofectamine 3000 (ThermoFisher).

Anchorage-independent growth in soft-agar was performed as previously described in (15). Briefly, $1 \times 10^{4}$ cells were plated in $0.35 \%$ Noble agar covered over a $0.5 \%$ agar bottom layer. Two or three weeks after plating, several random areas were imaged, and the colony number was quantified using the Image J software $(\mathrm{NIH})$.

All drugs, plasmids and antibodies used in this study are listed in Table S1.

\section{Tumor xenografts}

All xenograft experiments were approved and performed according to the KU Leuven IACUC guidelines (P117/2016). $2.5 \times 10^{5}$ of CH157-MN cells were injected subcutaneously in the flank of Rj:NMRI-Foxn1nu/nu mice. Tumor volumes were monitored every 2 days. The volume of each tumor 
was measured with a caliper using the formula: $V=\pi \times d 2 \times D / 6$, where $D$ is the major tumor axis and $d$ is the minor tumor axis.

\section{Analysis of protein expression, activity, and interactions}

Cells were washed in cold PBS and scraped on ice in the IP lysis buffer (50 mM Tris-HCl pH 7.5, 150 $\mathrm{mM} \mathrm{NaCl}, 1 \% \mathrm{NP}-40$ ) (Thermo Scientific) containing protease inhibitor and phosphatase inhibitor cocktails (Roche). Cell lysates were centrifugated for 10 minutes $16,000 \mathrm{~g}$ at $4^{\circ} \mathrm{C}$, and proteins were immunoprecipitated either using anti-Flag (M2) or anti-HA agarose beads (Sigma-Aldrich) overnight at $4^{\circ} \mathrm{C}$; or protein A/G magnetic beads (Thermo Scientific, \#88803) pre-mixed with primary antibody, or mouse IgG1 for 4 hours at $4^{\circ} \mathrm{C}$, and washed with ice-cold IP lysis buffer. Proteins were finally eluted with $3 \times$ Flag or HA peptides (Sigma-Aldrich) or by boiling for 10 minutes in 2x NuPAGE LDS sample buffer (Thermo Scientific, \#NP0007).

Ubiquitinated proteins were purified as described previously in (16). Subcellular fractionation was performed according to manufacturer's protocol (ThermoFisher). Protein stability in living cells was assessed by the global protein stability (GPS) approach $(17,18) .24$ hours after plating, HEK293T cells were transfected with the GPS reporter and shRNA constructs in a ratio 1:1. 48 hours after transfection cells were harvested and analyzed using the MACSQuant VYB flow cytometer (Miltenyi Biotec). Raw data were analyzed using FlowJo software (BD). Live single-cells were monitored for the expression of GFP and DsRed and the GFP/DsRed ratio were counted for measuring the relative protein stability. RAS activity was assessed using the RAS activation ELISA assay Kit (EMD Millipore). CDC42 activation assay was performed according to the manufacturer's protocol (Cytoskeleton, Inc). The proteomic and MS-based approaches are described in detail in Supplemental Material and Methods.

\section{Immunostaining and live imaging}


For immunostaining, $4 \times 10^{4}$ cells per well were plated on 8 -well chamber glass slide (Corning, \# 354118) and fixed with 4\% paraformaldehyde (PFA). Immunofluorescence was performed as described previously by (19). Briefly, cells were permeabilized in PBS-0.15\% Triton-X100 and then blocked with $1 \%$ BSA-10\% goat serum for 60 minutes. Primary antibodies, goat Alexa-conjugated secondary antibodies, Phalloidin-Atto 488 (Sigma-Aldrich, 49409). were diluted in blocking buffer and applied before final mounting with Vectashield (Vector Laboratories). Cross reactivity between antibodies was avoided by pre-blocking secondary anti-mouse antibodies with $10 \%$ rat serum. Images were captured using the Nikon $\mathrm{C} 2$ or the Nikon A1R confocal microscopes. Image analysis was performed using the software ImageJ (NIH).

For image acquisition of focal adhesions, a Nikon TiE inverted A1R (+ HD resonant scanning upgrade) microscope in combination with a 60x Oil objective (NA 1,4) The setup was controlled by NISElements (NIS 5.11.01 build 1368a, Nikon Instruments Europe B.V.). The number and size of the focal adhesions per cell was measured using a general analysis protocol (GA3) in NIS-elements (NIS 5.11.01 build 1368a, Nikon Instruments Europe B.V.). Individual cells were manually selected as regions of interest (ROIs). Within the ROIs, the focal adhesions were detected via a bright spot detection method after gaussian filtering of the image (kernel size 2). After removing artifacts, only focal adhesion sites inside the individual cells were measured. Live imaging is described in Supplemental Material and Methods.

\section{mRNA expression}

RNA extraction was performed using the NucleoSpin RNA kit (Machery Nagel). For RT-qPCR, 500ng of total RNA was used to prepare cDNA for mRNA analysis. cDNA was reverse-transcribed with the Sensifast cDNA synthesis kit (Bioline). Quantitative real-time PCR was performed with the LightCycler 
480 SYBR Green I Master reagent using the LightCycler 480 system (Roche). All primer sequences are reported in Table $\mathrm{S} 1$.

\section{DNA sequencing}

Original human tumor biopsies from benign meningioma were obtained fresh from the operating theater, and all patients gave written informed consent for use of their biopsy samples. All procedures involving human samples were approved by the UZ Leuven/KU Leuven medical ethics committee (ML8713/S54185). All procedures involving animals were approved and performed by the PDX platform in accordance with the guidelines of the KU Leuven IACUC (P147/2012). DNA extraction was performed on the PDX frozen tissues using DNeasy Blood \& Tissue Kit (Qiagen).

To detect KLF4 indels, genomic DNA of the cells was extracted using the NucleoSpin Tissue kit (Macherey-Nagel, \#740952), the loci encompassing gKLF4-1 (KLF4 exon3) and gKLF4-2 (KLF4 exon4) were amplified, and the amplicons were sequenced using their respective forward primers. The presence of InDels was determined using the Synthego ICE analysis tool (https://ice.synthego.com).

\section{Statistical and Bioinformatic analyses}

All data were analyzed using GraphPad Prism 8. Bioinformatic analyses are described in Supplemental Material and Methods

\section{Results}

\section{A multi-omics analysis reveals TRAF7-mediated alterations in human meningeal cells}

Some of the meningioma-associated mutations of TRAF7 present heterozygous indels (Figure 1A), suggesting that a partial loss of TRAF7 function could promote meningioma development. To model meningioma development, we used primary human meningeal cells (HMCs) immortalized by overexpressing the SV40 large T antigen. A partial suppression of TRAF7 in HMCs promoted their anchorage-independent (AI) growth, whereas overexpression of wt-TRAF7 in HMCs inhibited their 
ability to form soft agar colonies (Figure 1B,C; Figure S1A-D). In contrast, overexpression of the most recurrent meningioma-associated TRAF7 mutants, TRAF7-T391I, TRAF7-N520S, and TRAF7-G536S, or the truncated TRAF7-E353* mutant did not affect the AI growth (Figure 1C; Figure S1C,D). Similarly, ectopic expression of wt-TRAF7 inhibited the AI growth of meningioma CH157-MN cells (Figure S1E). Furthermore, suppression of TRAF7 in CH157-MN cells promoted the xenograft growth (Figure 1D,E; Figure S1F). These results indicate a tumor suppressive function of TRAF7 in meningeal cells.

To uncover the molecular mechanism by which TRAF7 contributes to meningioma development, we interrogated TRAF7-mediated alterations in meningeal cells by integrating ubiquitinome, proteome, and transcriptome analyses (Figure 1F; Figure S1G). In parallel, we characterized TRAF7 interactome by employing a mass spectrometry (MS)-based Virotrap approach, which allows trapping protein complexes from intact mammalian cells under native conditions (20) (Figure 1F). To identify the functional biological processes modulated by TRAF7, we performed an Ingenuity Pathway Analysis (IPA) in all datasets. We found that the ubiquitome and proteome datasets shared most of the top ranked pathways, but showed a limited overlap with the pathways altered at mRNA level (Figure 1G, Figure S2). Thus, a multi-omics analysis of meningeal cells revealed a global TRAF7-mediated proteome rewiring, with the majority of dynamic protein expression being regulated posttranslationally.

Integrating TRAF7-driven alterations and TRAF7 interactome point out the role of TRAF7 in the regulation of actin cytoskeleton (Figure 1G; Figure 2A). Consistently, we found that TRAF7 is localized in the cytoplasm and at the leading edge of meningeal cells (Figure 2B). Moreover, immunostaining analysis of HMCs showed co-localization of TRAF7 with actin cytoskeleton at the leading edges, but no co-localization with tubulin (Figure S3A). Next, we examined the effect of TRAF7 on the dynamics of the actin cytoskeleton. Time-lapse video of HMCs showed that TRAF7 suppression promoted actin 
dynamics, inducing contraction, retraction, and protrusions at both leading and rear edges (Figure 2C; Movie S1,S2). TRAF7 depletion also led to an increased number of filopodia and slowed down filopodial tip retraction, hence enhanced stability of non-stationary filopodia (Figure 2C,D). More stable filopodia in TRAF7-depleted cells were associated with an increased number of focal adhesion (Figure 2E). In contrast, wt-TRAF7 suppressed actin dynamics (Figure S3B; Movie S3,S4). We observed decreased filopodia number and stability as well as a reduced number of focal adhesions in HMCs overexpressing wt-TRAF7 (Figure 2F, G). Altogether, these results implicate TRAF7 in the regulation of actin dynamics. Similarly, the proteomic analysis of NF2-mutated meningiomas revealed focal adhesion and actin cytoskeleton signaling among the most significantly enriched pathways (21). This strongly indicates that mutations of both $N F 2$ and TRAF7 converge on alterations of the actin cytoskeleton.

\section{TRAF7 interacts with RAS-related GTPases}

The Actin cytoskeleton pathway was among the top 10 pathways enriched in the TRAF7 interactome (Figure 2A, Figure S2). This pathway was also highly enriched among potential TRAF7 interactors identified by the BioPlex network $\left(\mathrm{p}=6.29 \mathrm{e}^{-3}\right)(22)$. Among putative TRAF7 interactors involved in the regulation of actin cytoskeleton, we identified several RAS-related GTPases, such as Cell division cycle 42 (CDC42), Kirsten Rat Sarcoma Viral Oncogene Homolog (KRAS), Harvey Rat Sarcoma Viral Oncogene Homolog (HRAS), RAC Family Small GTPase 1/2/3 (RAC1/RAC2/RAC3), Related RAS Viral Oncogene Homolog 2 (RRAS2), and RAS Homolog Family Member A (RHOA) (Figure 3A). For further studies, we decided to focus on CDC42, a key regulator of filopodia formation, and on the RAS proto-oncogenes. Activating mutations in $C D C 42$ were found be to mutually exclusive with TRAF7 mutations in differentiated papillary mesotheliomas (23), suggesting that TRAF7 and CDC42 might belong to the same signaling network. On the other hand, we previously identified TRAF7 as a potential 
HRAS interactor in a Virotrap screen, in which HRAS served as bait (19). Moreover, germline TRAF7 mutations in the TRAF7 Syndrome patients phenotypically overlap with RASopathy, a group of genetic syndromes caused by changes in genes that are part of the RAS pathway $(24,25)$.

To confirm the interaction between TRAF7 and RAS proteins and CDC42, we performed a set of immunoprecipitations. We found that Flag-tagged TRAF7 coimmunoprecipitated with endogenous RAS proteins and CDC42 (Figure 3B, C). Reciprocal coimmunoprecipitations demonstrated that TRAF7 interacted with all RAS isoforms and CDC42 (Figure 3C-E). Moreover, immunostainings analysis showed co-localization of TRAF7 with NRAS and CDC42, mostly at the leading edge of meningeal cells (Figure 3F). These results indicate that TRAF7 forms a complex with small GTPases, RAS and CDC42.

Given that the majority of recurrent TRAF7 mutations in meningioma are located within the WD40 repeat domain responsible for the substrate recognition (13) (Figure 1A), we assessed whether these mutations affect the ability of TRAF7 to bind RAS and/or CDC42 (Figure 3G). We found that the truncated TRAF7-E353* and the most recurrent WD40 domain TRAF7 mutants showed reduced binding to either NRAS, or CDC42. On the other hand, the TRAF7-R153S mutation located in the RING catalytic domain, which we identified in our cohort of meningioma patients, did not affect binding to NRAS or CDC42 (Figure 1A, Figure 3G). The results suggest that TRAF7 mutations in the WD40 domain could promote meningioma development by disrupting TRAF7 binding to the RAS-like GTPases.

\section{TRAF7 loss of function promotes the anchorage-independent growth of human meningeal cells}

The ubiquitome analysis revealed that TRAF7 suppression decreased CDC42 ubiquitination at different sites with the most pronounced effect at K163 (Figure 4A). We also observed a tendency to lower levels 
of ubiquitination of several RAS-related GTPases in TRAF7-depleted HMCs (Figure 4A). His-ubiquitin pull-down confirmed that TRAF7 suppression led to decreased ubiquitination of NRAS (Figure 4B; Figure S4A). In contrast, overexpression of wt-TRAF7, but not meningioma-associated TRAF7 mutants, increased ubiquitination of both CDC42 and NRAS (Figure 4C,D). These results indicate that TRAF7 controls ubiquitination of several RAS-related GTPases, while meningioma-associated TRAF7 mutants lose this function.

The proteome analysis demonstrated that suppression of TRAF7 slightly increased expression of the RAS-related GTPases (Figure 4E). Immunoblotting corroborated increased protein expression of panRAS and CDC42 in TRAF7-depleted HMCs, whereas we did not observe an increase of RAS-related GTPases at mRNA level (Figure 4F, 4G). This suggests that TRAF7 could regulate the protein stability of RAS-like small GTPases. To test this idea, we utilized the global protein stability (GPS) approach, a fluorescence-based system for monitoring protein stability in living cells $(17,18)$. We generated RAS and CDC42 stability reporter systems that contain DsRed followed by an internal ribosome entry site 2 (IRES2) and GFP-fused RAS GTPases (Figure S4B). The increase in GFP-RAS signals positively correlated with the presence of the proteasome inhibitor MG132, whereas the DsRed signal remained constant, indicating that the GFP/DsRed ratio reflects the stability of the RAS-related GTPases (Figure S4C). The GPS analysis showed that TRAF7 suppression led to increased stability of all RAS isoforms and CDC42 (Figure 4H). Previous studies also demonstrated that ubiquitination of the RAS GTPases affects their subcellular localization (19). Consistent with these reports, we found that, in addition to increasing the stability of the RAS-related GTPases, TRAF7 depletion also led to an accumulation of RAS and CDC42 in the membrane fraction, indicating that TRAF7 controls subcellular localization of RAS and CDC42 (Figure 4I). Altogether, these results implicate TRAF7 in the proteostatic regulation of the RAS-related small GTPases. 
Live imaging implicated TRAF7 in regulating the dynamic of the actin cytoskeleton (Figure 2C-G; Figure S3B), suggesting that TRAF7-mediated ubiquitination alters the activity of the RAS-like GTPases. Consistently, we found that loss of TRAF7 in meningeal cells promoted the activity of CDC42 and p21-activated kinase (PAK1) (Figure 5A,B, Figure S4D). TRAF7 knockdown in HMCs or CH157MN cells also led to enhanced RAS activity and up-regulation of the MAPK cascade (Figure 5C,D, Figure S4E,F). In contrast, overexpression of wt-TRAF7, but not TRAF7 mutants, suppressed the activity of the CDC42/PAK and RAS/MAPK signaling cascades (Figure 5E-G; Figure S4G,H). Altogether, these results indicate that TRAF7 loss of function induces the activity of both CDC42/PAK and RAS/MAPK pathways.

We next validated the contribution of TRAF7-mediated ubiquitination of the RAS-like GTPases to tumorigenic transformation of HMCs. We found that either the ubiquitination-deficient CDC42-K163R, or the constitutively active CDC42-G12V mutant promoted the AI growth of HMCs (Figure 4A, Figure 5H). Moreover, the PAK inhibitor FRAX597 diminished AI growth of TRAF7-depleted HMCs, but did not affect the growth of HMCs expressing shGFP, indicating that activation of the CDC42/PAK pathway could contribute to TRAF7-mediated cell transformation (Figure 5I).

We also generated ubiquitination-deficient NRAS (UbDef-NRAS) by mutating 5 lysine residues, K104, K117, K128, K135, and K147, found to be ubiquitinated in the MS analyses. Overexpression of the ubiquitination-deficient NRAS mutant promoted the AI growth of HMCs expressing sh $G F P$, whereas the UbDef-NRAS mutant did not significantly increase the growth of TRAF7-depleted HMCs (Figure $5 \mathrm{~J}$ ), suggesting that dysregulation of RAS ubiquitination could contribute to transformation driven by TRAF7 loss. On the other hand, the oncogenic NRAS-Q61K mutant suppressed the ability of HMCs to form colonies in soft agar (Figure 5K). Together, these results suggest that up-regulation of the RAS pathway might have a dual effect on tumorigenic transformation of HMCs. 


\section{TRAF7 loss promotes the tumor suppressive function of KLF4}

Inhibition of the AI growth of meningeal cells by the oncogenic RAS (Figure 5K) suggests that TRAF7mediated activation of RAS/MAPK signaling may turn on tumor suppressive mechanisms. Given that KLF4 mutations are observed in $40 \%$ of TRAF7 mutated meningiomas and KLF4 expression is upregulated by the RAS/MAPK pathway $(26,27)$, we hypothesized that TRAF7 loss might promote the tumor suppression function of KLF4.

Consistently, we found an increased KLF4 expression in TRAF7 mutated meningiomas compared to adult meninges and non-TRAF7 mutated meningiomas (Figure 6A). TRAF7 depletion in HMCs or CH157-MN cells also led to increased KLF4 expression (Figure 6B,C; Figure S5A,B). Furthermore, the IPA upstream regulator analysis predicted KLF4 activation in TRAF7-depleted HMCs (activation zscore=2.074). Inhibition of the MAPK pathway by the MEK inhibitor pimasertib abolished TRAF7mediated KLF4 up-regulation (Figure 6D; Figure S5B). Moreover, KLF4 expression was induced by the NRAS-Q61K mutant, but not by the constitutively active CDC42-G12V mutant (Figure 6E). These results indicate that KLF4 upregulation is triggered by TRAF7-mediated activation of the RAS/MAPK pathway.

The most well-described KLF4 transcriptional target involved in growth inhibition is Cyclin Dependent Kinase Inhibitor 1A $(C D K N 1 A)(28)$. However, wt-KLF4 overexpression in meningeal cells did not induce CDKNIA expression, suggesting KLF4 regulates CDKNIA expression in a tissue-specific manner (Figure S5C). To elucidate the tumor-suppressive mechanism of KLF4 during meningioma development, we searched for KLF4 target genes up-regulated in TRAF7-depleted or wt-KLF4overexpressing HMCs and down-regulated in KLF4-depleted HBL52 cells. HBL52 is the benign meningioma cell line harboring TRAF7-G536S mutation and wt-KLF4 (29) (Figure S5D). Among the identified KLF4 target genes, Semaphorin 3F (SEMA3F) and Cellular Retinoic Acid Binding Protein 2 
$(C R A B P 2)$ were dysregulated in all three datasets (Figure 6F). RT-qPCR analysis confirmed the upregulation of $C R A B P 2$ and SEMA3F by wt-KLF4 overexpression or TRAF7 suppression in HMCs (Figure 6G,H). In contrast, KLF4 knockdown in HBL52 cells decreased expression of CRABP2 and SEMA3F (Figure 6I). Moreover, overexpression of SEMA3F, but not CRABP2, inhibited the AI growth of meningeal cells (Figure 6J). These results indicate that TRAF7-mediated up-regulation of KLF4 might suppress meningioma development by inducing the Semaphorin Pathway. The Semaphorin pathway is an indispensable regulator of neuron-axonal guidance (30), whereas Axonal Guidance Signaling was highly enriched in our model systems as well as in double KLF4/ TRAF7 mutant meningiomas compared with wt-KLF4/mutant TRAF7 meningiomas (Figure 6K, Figure S5E). The Axonal Guidance pathway was also the top enriched pathway in TRAF7-mutated fibroblasts of the TRAF7 syndrome patients (25) and in the proteome analysis of TRAF7-depleted HMCs (Figure 1G, Figure S2), further confirming the contribution of Semaphorin signaling in meningioma development. Given that Semaphorin signaling suppresses the activity of the RAS and RHO family members, TRAF7-mediated activation of the Semaphorin pathway might represent a negative feedback loop to fine tune the GTPase activity.

\section{TRAF7 and KLF4 cooperate to promote transformation of human meningeal cells}

We next explored the cooperation between TRAF7 and KLF4 to promote meningioma development. TRAF7-mutated meningiomas harbor a recurrent $K L F 4-K 409 Q$ mutation, which exclusively occurs in meningiomas with extremely rare occurrences in other tumors. K409 residue in the first zinc finger domain is involved in direct contact with DNA (Figure 1A) (31). In silico modeling indicated that K409Q mutations might disrupt KLF4 interaction with DNA (Figure S6A), suggesting that K409Q mutation is a loss of function. The IPA upstream regulator analysis also predicted upregulation of KLF4 activity in HMCs overexpressing wt-KLF4, but not the KLF4-K409Q mutant (Figure 6L, Figure S5C). 
KLF4-K409Q did not also induce expression of SEMA3F (Figure 6G). Furthermore, overexpression of wt-KLF4 suppressed AI growth of HMCs, whereas the KLF4-K409Q mutant slightly promoted the ability of HMCs to grow in soft agar (Figure 6L).

Loss of function KLF4 mutation might abrogate the tumor suppressive function of wt-KLF4 induced by TRAF7 loss. Concordantly, KLF4 loss in TRAF7-depleted HMCs or TRAF7-mutated HBL52 cells promoted their AI growth, whereas such effect was not in HMCs with intact TRAF7 expression (Figure 6M-O, Figure S6C-F). Moreover, we observed a cooperation between loss of TRAF7 and KLF4 in short-term cultured meningioma CH157-MN cells. Whereas suppression of either KLF4 or TRAF7 did not dramatically affect their growth in soft agars, suppression of both KLF4 and TRAF7 induced AI growth (Figure 6P; Figure S7A,B). Overexpression of either TRAF7, or KLF4 partially rescued the increased AI growth of HMCs expressing a combination of shTRAF7 and shKLF4 (Figure S7C,D). Together, these results indicate that KLF4 loss-of-function break the Semaphorin-induced inhibition loop down and cooperates with the loss of TRAF7 to enhance tumorigenic transformation of meningeal cells. 


\section{Discussion}

Recent advances in next-generation sequencing have uncovered the mutational signature of meningiomas. Proteomic profiling and functional studies are the next critical steps in advancing our understanding of meningioma biology. Integrative analysis of TRAF7 loss-of-function in human meningeal cells demonstrated that dysregulation of TRAF7 activity leads to alterations in actin cytoskeleton at the posttranslational level due to the activation of RAS-related GTPases. Even though early studies linked TRAF7 to the modulation of the Nuclear factor-kB transcription factor and TNF $\alpha$ mediated activation of cellular stress pathways (32), TRAF7 lacks the TRAF homology domain responsible for its association with the cytoplasmic regions of receptors of the TNF-R superfamily. Instead of the TRAF domain, the C-terminus of TRAF7 contains the WD40 repeats domain. The WD40 domain was reported to have a scaffolding structure known to be involved in the interaction with small GTPases $(33,34)$.

Similar to TRAF7-depleted meningeal cells, NF2-deficient meningioma cells also show dramatic differences in actin cytoskeletal organization (35). The NF2 protein Merlin, an ezrin-moesin-radixin family member, is a tumor suppressor that links the actin cytoskeleton with plasma membrane proteins and mediate contact-dependent cell growth inhibition. Emerging evidences indicate the interconnection between Merlin and RAS-related GTPases. RAC and CDC42 induce PAK-mediated inactivating phosphorylation of Merlin (36), whereas Merlin inhibits the activity of the RHO-related GTPases and modulates the activity of RAS signaling (37). These functional interactions between Merlin and the RAS-like GTPases indicate a feed-forward mechanism for ensuring their coordinated activation and inactivation. This strongly indicates that mutations of both NF2 and TRAF7 converge on hyperactivation of RAS-related GTPases and alterations of the actin cytoskeleton. 
TRAF7 loss-of-function leads to dysregulation of proteostasis and activation of several RAS-related small GTPases, such as CDC42 and all RAS isoforms. Previous studies demonstrated that increased RAS stability could drive oncogenic transformation. Higher stability of RAS proteins in colorectal cancer could be due to decreased expression of the E3 ubiquitin ligase NEDD4(38) or dysregulation of $\beta$-TrCP-mediated RAS degradation in APC-mutated tumors $(39,40)$. Another proteostatic regulator of RAS proteins is the leucine zipper-like transcriptional regulator 1 (LZTR1)/CUL3 ubiquitin ligase complex that controls localization and expression levels of RAS proteins (19,41). LZTR1 mutations are associated with Noonan Syndrome, glioblastoma and schwannomatosis (42-46). Interestingly, the TRAF7 Syndrome resulting from germline TRAF7 mutations phenotypically overlaps with RASopathy disorders $(24,25)$.

On the other hand, dysregulation of X-linked inhibitor of apoptosis protein (XIAP)-dependent CDC42 ubiquitination enhances filopodia formation and promotes lung colonization of tumor cells in mice in a Cdc42-dependent manner (47). Of note, activating mutations in CDC42 are mutually exclusive with TRAF7 mutations in differentiated papillary mesotheliomas (23), suggesting that TRAF7 and CDC42 could act within the same signaling network. Altogether, these studies highlight the importance of ubiquitination in the regulation of small GTPase.

Whereas activation of CDC42 in meningeal cells promotes AI growth, RAS activation leads to paradoxical growth inhibition. RAS-mediated induction of KLF4 and activation of Semaphorin signaling appear to be responsible for this growth inhibition. Semaphorins have been found to inhibit proliferation and metastatic spread of tumor cells as well as suppress tumor progression by modulating tumor microenvironment $(48,49)$. Semaphorins transduce the signal through their interaction with members of the plexin and neuropilin families of transmembrane receptors. A hallmark of Semaphorin receptors is their ability to interact directly with small GTPases of the RAS and RHO families through 
their intracellular region and to act as GTPase activating proteins (GAPs). This suggests that TRAF7mediated activation of the Semaphorin pathway might represent a negative feedback loop to fine tune the GTPase activity, whereas KLF4 mutation might breakdown this feedback to fully activate small GTPases.

The emerging molecular knowledge of meningioma development provides a tremendous opportunity to explore novel therapeutic strategies for meningioma patients. Our results suggest that treating meningiomas with MEK or BRAF inhibitors could moderate the tumor suppressive function of KLF4. On the other hand, the use of PAK inhibitors, such as FRAX597, could be beneficial for treating TRAF7-mutated meningiomas. PAKs have increasingly attracted attention as potential therapeutic targets due to their central roles in oncogenic signaling pathways. Both pan-PAK inhibitors and groupspecific PAK inhibitors have been extensively tested for their anti-cancerous efficacy in different cancer models(50). Thus, integrated omics studies that outline clinically important genetic changes of meningioma will facilitate comprehensive and personalized care of meningioma patients.

\section{Author contribution}

PN performed biochemical, cellular and immunostaining experiments; SP performed experiments most of using CH157-MN cells; NC assessed CRISPR-mediated alterations; MS performed immunoprecipitation experiments and ubiquitination pull-downs; DVH, TNM, and FI performed the MS experiments; SE, performed the Virotrap Screen; MFB performed the GPS experiments; BL performed RNA extractions; RNS performed xenograft experiments; TNM and PZ performed bioinformatic analysis; NC generated pipelines for image analysis; AAS designed the study; PN, and AAS analyzed the data and wrote the manuscript. All authors discussed the results and commented on the manuscript. 


\section{References}

1. Bi WL, Zhang M, Wu WW, Mei Y, Dunn IF. Meningioma Genomics: Diagnostic, Prognostic, and Therapeutic Applications. Front Surg 2016;3:40

2. Preusser M, Brastianos PK, Mawrin C. Advances in meningioma genetics: novel therapeutic opportunities. Nat Rev Neurol 2018; $14: 106-15$

3. Yuzawa S, Nishihara H, Tanaka S. Genetic landscape of meningioma. Brain Tumor Pathol 2016;33:237-47

4. Zotti T, Scudiero I, Vito P, Stilo R. The Emerging Role of TRAF7 in Tumor Development. J Cell Physiol 2017;232:1233-8

5. Klein CJ, Wu Y, Jentoft ME, Mer G, Spinner RJ, Dyck PJ, et al. Genomic analysis reveals frequent TRAF7 mutations in intraneural perineuriomas. Ann Neurol 2017;81:316-21

6. Goode B, Joseph NM, Stevers M, Van Ziffle J, Onodera C, Talevich E, et al. Adenomatoid tumors of the male and female genital tract are defined by TRAF7 mutations that drive aberrant NF-kB pathway activation. Mod Pathol 2018;31:660-73

7. Bueno R, Stawiski EW, Goldstein LD, Durinck S, De Rienzo A, Modrusan Z, et al. Comprehensive genomic analysis of malignant pleural mesothelioma identifies recurrent mutations, gene fusions and splicing alterations. Nat Genet 2016;48:407-16

8. Hung YP, Dong F, Dubuc AM, Dal Cin P, Bueno R, Chirieac LR. Molecular characterization of localized pleural mesothelioma. Mod Pathol 2020;33:271-80

9. Zammarchi F, Morelli M, Menicagli M, Di Cristofano C, Zavaglia K, Paolucci A, et al. KLF4 is a novel candidate tumor suppressor gene in pancreatic ductal carcinoma. Am J Pathol 2011; $178: 361-72$

10. Yu F, Li J, Chen H, Fu J, Ray S, Huang S, et al. Kruppel-like factor 4 (KLF4) is required for maintenance of breast cancer stem cells and for cell migration and invasion. Oncogene 2011;30:2161-72

11. Yu T, Chen X, Zhang W, Liu J, Avdiushko R, Napier DL, et al. KLF4 regulates adult lung tumor-initiating cells and represses K-Ras-mediated lung cancer. Cell Death Differ 2016;23:20715

12. Tang H, Zhu H, Wang X, Hua L, Li J, Xie Q, et al. KLF4 is a tumor suppressor in anaplastic meningioma stem-like cells and human meningiomas. J Mol Cell Biol 2017;9:315-24 
13. Clark VE, Erson-Omay EZ, Serin A, Yin J, Cotney J, Ozduman K, et al. Genomic analysis of non-NF2 meningiomas reveals mutations in TRAF7, KLF4, AKT1, and SMO. Science

2013;339:1077-80

14. Clark VE, Harmancı AS, Bai H, Youngblood MW, Lee TI, Baranoski JF, et al. Recurrent somatic mutations in POLR2A define a distinct subset of meningiomas. Nat Genet

2016;48:1253-9

15. Boehm JS, Zhao JJ, Yao J, Kim SY, Firestein R, Dunn IF, et al. Integrative genomic approaches identify IKBKE as a breast cancer oncogene. Cell 2007;129:1065-79

16. Simicek M, Lievens S, Laga M, Guzenko D, Aushev VN, Kalev P, et al. The deubiquitylase USP33 discriminates between RALB functions in autophagy and innate immune response. Nat Cell Biol 2013;15:1220-30

17. Yen HC, Xu Q, Chou DM, Zhao Z, Elledge SJ. Global protein stability profiling in mammalian cells. Science 2008;322:918-23

18. Yen HC, Elledge SJ. Identification of SCF ubiquitin ligase substrates by global protein stability profiling. Science 2008;322:923-9

19. Steklov M, Pandolfi S, Baietti MF, Batiuk A, Carai P, Najm P, et al. Mutations in LZTR1 drive human disease by dysregulating RAS ubiquitination. Science 2018;362:1177-82

20. Eyckerman S, Titeca K, Van Quickelberghe E, Cloots E, Verhee A, Samyn N, et al. Trapping mammalian protein complexes in viral particles. Nat Commun 2016;7:11416

21. Bassiri K, Ferluga S, Sharma V, Syed N, Adams CL, Lasonder E, et al. Global Proteome and Phospho-proteome Analysis of Merlin-deficient Meningioma and Schwannoma Identifies PDLIM2 as a Novel Therapeutic Target. EBioMedicine 2017;16:76-86

22. Huttlin EL, Bruckner RJ, Paulo JA, Cannon JR, Ting L, Baltier K, et al. Architecture of the human interactome defines protein communities and disease networks. Nature 2017;545:505-9

23. Stevers M, Rabban JT, Garg K, Van Ziffle J, Onodera C, Grenert JP, et al. Well-differentiated papillary mesothelioma of the peritoneum is genetically defined by mutually exclusive mutations in TRAF7 and CDC42. Mod Pathol 2019;32:88-99

24. Tokita MJ, Chen CA, Chitayat D, Macnamara E, Rosenfeld JA, Hanchard N, et al. De Novo Missense Variants in TRAF7 Cause Developmental Delay, Congenital Anomalies, and Dysmorphic Features. Am J Hum Genet 2018;103:154-62 
25. Castilla-Vallmanya L, Selmer KK, Dimartino C, Rabionet R, Blanco-Sánchez B, Yang S, et al. Phenotypic spectrum and transcriptomic profile associated with germline variants in TRAF7. Genet Med 2020;22:1215-26

26. Kim MO, Kim SH, Cho YY, Nadas J, Jeong CH, Yao K, et al. ERK1 and ERK2 regulate embryonic stem cell self-renewal through phosphorylation of Klf4. Nat Struct Mol Biol 2012; $19: 283-90$

27. Riverso M, Montagnani V, Stecca B. KLF4 is regulated by RAS/RAF/MEK/ERK signaling through E2F1 and promotes melanoma cell growth. Oncogene 2017;36:3322-33

28. Rowland BD, Peeper DS. KLF4, p21 and context-dependent opposing forces in cancer. Nat Rev Cancer 2006;6:11-23

29. Mei Y, Bi WL, Greenwald NF, Agar NY, Beroukhim R, Dunn GP, et al. Genomic profile of human meningioma cell lines. PLoS One 2017;12:e0178322

30. Kolodkin AL, Matthes DJ, Goodman CS. The semaphorin genes encode a family of transmembrane and secreted growth cone guidance molecules. Cell 1993;75:1389-99

31. Schuetz A, Nana D, Rose C, Zocher G, Milanovic M, Koenigsmann J, et al. The structure of the Klf4 DNA-binding domain links to self-renewal and macrophage differentiation. Cell Mol Life Sci 2011;68:3121-31

32. Zotti T, Vito P, Stilo R. The seventh ring: exploring TRAF7 functions. J Cell Physiol 2012;227:1280-4

33. Schapira M, Tyers M, Torrent M, Arrowsmith CH. WD40 repeat domain proteins: a novel target class? Nat Rev Drug Discov 2017;16:773-86

34. Shen W, Kilander MBC, Bridi MS, Frei JA, Niescier RF, Huang S, et al. Tomosyn regulates the small RhoA GTPase to control the dendritic stability of neurons and the surface expression of AMPA receptors. J Neurosci Res 2020;98:1213-31

35. James MF, Lelke JM, Maccollin M, Plotkin SR, Stemmer-Rachamimov AO, Ramesh V, et al. Modeling NF2 with human arachnoidal and meningioma cell culture systems: NF2 silencing reflects the benign character of tumor growth. Neurobiol Dis 2008;29:278-92

36. McClatchey AI. Merlin and ERM proteins: unappreciated roles in cancer development? Nat Rev Cancer 2003;3:877-83 
37. Cui Y, Groth S, Troutman S, Carlstedt A, Sperka T, Riecken LB, et al. The NF2 tumor suppressor merlin interacts with Ras and RasGAP, which may modulate Ras signaling. Oncogene 2019;38:6370-81

38. Zeng T, Wang Q, Fu J, Lin Q, Bi J, Ding W, et al. Impeded nedd4-1-mediated ras degradation underlies ras-driven tumorigenesis. Cell Rep 2014;7:871-82

39. Kim SE, Yoon JY, Jeong WJ, Jeon SH, Park Y, Yoon JB, et al. H-Ras is degraded by Wnt/betacatenin signaling via beta-TrCP-mediated polyubiquitylation. J Cell Sci 2009;122:842-8

40. Moon BS, Jeong WJ, Park J, Kim TI, Min dS, Choi KY. Role of oncogenic K-Ras in cancer stem cell activation by aberrant Wnt/ $\beta$-catenin signaling. J Natl Cancer Inst 2014;106:djt373

41. Bigenzahn JW, Collu GM, Kartnig F, Pieraks M, Vladimer GI, Heinz LX, et al. LZTR1 is a regulator of RAS ubiquitination and signaling. Science 2018;362:1171-7

42. Paganini I, Chang VY, Capone GL, Vitte J, Benelli M, Barbetti L, et al. Expanding the mutational spectrum of LZTR1 in schwannomatosis. Eur J Hum Genet 2015;23:963-8

43. Piotrowski A, Xie J, Liu YF, Poplawski AB, Gomes AR, Madanecki P, et al. Germline loss-offunction mutations in LZTR1 predispose to an inherited disorder of multiple schwannomas. Nat Genet 2014;46:182-7

44. Smith MJ, Isidor B, Beetz C, Williams SG, Bhaskar SS, Richer W, et al. Mutations in LZTR1 add to the complex heterogeneity of schwannomatosis. Neurology 2015;84:141-7

45. Yamamoto GL, Aguena M, Gos M, Hung C, Pilch J, Fahiminiya S, et al. Rare variants in SOS2 and LZTR1 are associated with Noonan syndrome. J Med Genet 2015;52:413-21

46. Johnston JJ, van der Smagt JJ, Rosenfeld JA, Pagnamenta AT, Alswaid A, Baker EH, et al. Autosomal recessive Noonan syndrome associated with biallelic LZTR1 variants. Genet Med 2018;20:1175-85

47. Murali A, Shin J, Yurugi H, Krishnan A, Akutsu M, Carpy A, et al. Ubiquitin-dependent regulation of Cdc42 by XIAP. Cell Death Dis 2017;8:e2900

48. Capparuccia L, Tamagnone L. Semaphorin signaling in cancer cells and in cells of the tumor microenvironment--two sides of a coin. J Cell Sci 2009;122:1723-36

49. Neufeld G, Mumblat Y, Smolkin T, Toledano S, Nir-Zvi I, Ziv K, et al. The role of the semaphorins in cancer. Cell Adh Migr 2016;10:652-74

50. Radu M, Semenova G, Kosoff R, Chernoff J. PAK signalling during the development and progression of cancer. Nat Rev Cancer 2014; 14:13-25 


\section{Figure legends}

Figure 1. Integrated analysis of TRAF7-mediated alterations of ubiquitome, proteome and transcriptome and TRAF7 interactome. A, Schematic diagram of the TRAF7 and KLF4 domains showing the mutated residues in meningiomas; mutations analyzed in this study are indicated. RING, the ubiquitin ligase RING catalytic domain; WD40, WD40 repeats domain; ZNF, zinc finger domain. B, AI growth of HMCs expressing shRNA targeting GFP or TRAF7. Values are means $\pm \mathrm{SEM}$; $\geq 3$; $\mathrm{P}$ values are calculated by two-sided Student's t test. C, Immunoblotting analysis and AI growth of HMCs expressing an empty vector (EV), wt-TRAF7, or the indicated TRAF7 mutants. Values are means \pm SEM; $\mathrm{n}=3 ; \mathrm{P}$ values are calculated by one-way ANOVA. D, Growth of CH157-MN xenografts expressing shGFP or shTRAF7-2. n=6; P value was assessed by two-way ANOVA. E, Tumor weight of CH157-MN xenografts expressing shGFP or shTRAF7-2 at the end point is presented as mean \pm SEM, n=6 per group. $\mathrm{P}$ value was assessed by Mann-Whitney unpaired t-test. F, A workflow for studying TRAF7-mediated alterations and the TRAF7 interactome. G, Ingenuity Pathway Analysis of TRAF7mediated alterations and TRAF7 interactome. Top 10 significantly enriched pathways for each of the datasets are visualized as a network using the EnrichmentMap Cytoscape App. The piecharts indicate datasets that showed significant pathway enrichment $(\mathrm{P}<0.01)$ of the indicated canonical pathways. The pathways shared the same genes are connected by lines.

Figure 2. TRAF7 is a regulator of cytoskeleton organization. A, Overall enrichment scores of the canonical IPA pathways identified across all datasets shown in Figure 1F,G. B, Immunostaining of HMCs expressing HA-tagged TRAF7 with anti-HA antibody. Scale bar, $10 \mu \mathrm{m}$. C, Representative still images of live imaging of actin dynamics in HMCs expressing shGFP or shTRAF7. Acquisition every 2 minutes for 10 minutes. Scale bar, $10 \mu \mathrm{m}$. D, Actin dynamics in HMCs expressing shGFP or shTRAF7. The number of filopodia per cell is shown as a whisker plot. $\mathrm{P}$ values were calculated by a two-sided 
Student's $\mathrm{t}$ test. The filopodia length is shown as mean \pm SEM; P values were detected by two-way ANOVA. E, Immunostaining of HMCs expressing shGFP or shTRAF7 with anti-vinculin antibody. Scale bar, $10 \mu \mathrm{m}$. The number of focal adhesions per cells is shown as a whisker plot. $\mathrm{P}$ values were calculated by a two-sided Student's t test. F, Imaging of actin dynamics in HMCs expressing an empty vector $(\mathrm{EV})$ or HA-TRAF7. The filopodia length is shown as mean $\pm \mathrm{SEM}$; P values were detected by two-way ANOVA. The number of filopodia per cell is shown as a whisker plot. P values were calculated by a two-sided Student's t test. G, Immunostaining of HMCs expressing an empty vector (EV) or HA-TRAF7 with anti-vinculin antibody. Scale bar, $10 \mu \mathrm{m}$. The number of focal adhesions per cells is shown as mean \pm SEM; $P$ values were calculated by a two-sided Student's $t$ test. B,D,E,F,G, Number of analyzed cells $\geq 50$. A whisker plot depicting the 5th, 25th, 75th, and 95th percentiles with outliers.

Figure 3. TRAF7 forms a complex with the RAS-related GTPases. A, A Virotrap screen performed in HEK293T cells using group-specific antigen (GAG)-TRAF7 as bait. Escherichia coli dihydrofolate reductase (eDHFR) fused to GAG was used as a negative control. A heatmap showing the RAS-related GTPases differentially interacting with eDHFR and TRAF7. The scale shows Z-scored site intensity values. B, Flag-tagged TRAF7 was immunoprecipitated from HEK293T cell lysates using anti- FLAG (M2) resin. CDC42 and panRAS were detected by immunoblotting. C, panRAS or Flag-tagged CDC42 was immunoprecipitated from HEK293T cells overexpressing FLAG-tagged or HA-tagged TRAF7 using anti-panRAS antibody coupled to protein A/G agarose resin or anti-FLAG (M2) resin. Flag or HA-tagged TRAF7 was detected by immunoblotting. D, HA-tagged RAS proteins were immunoprecipitated from HEK293T cells overexpressing Flag-tagged TRAF7 using anti-HA resin. TRAF7 was detected by immunoblotting with anti-Flag-antibody. E, Flag-tagged TRAF7 was immunoprecipitated from HEK293T cells overexpressing HA-tagged RAS using anti-FLAG (M2) resin. 
RAS isoforms were detected by immunoblotting with anti-HA antibody. F, Immunostaining of HMCs expressing HA-TRAF7 and mCherry-CDC42 or mCherry-NRAS with anti-HA antibody. Scale bar, 10 $\mu \mathrm{m}$. G, HA-tagged CDC42 or RAS proteins were immunoprecipitated from HEK293T cells overexpressing Flag-tagged TRAF7 using anti-HA resin. TRAF7 was detected by immunoblotting with anti-Flag-antibody.

Figure 4. TRAF7 controls proteostasis of the RAS-related GTPases. A, A heatmap showing the change in ubiquitination of RAS-related GTPases in HMCs expressing shGFP or shTRAF7. The scale shows $\log 2$ (fold of change) of site intensity values. n=3 B, Ubiquitinated Flag-tagged NRAS was purified from HEK293T cells expressing the indicated constructs by $\mathrm{Co}^{2+}$ metal affinity chromatography and detected by immunoblotting. C, Ubiquitinated Flag-tagged CDC42 was purified from HEK293T cells expressing Flag-tagged wt-TRAF7 or TRAF7 mutants by $\mathrm{Co}^{2+}$ metal affinity chromatography and detected by immunoblotting. D, Ubiquitinated Flag-tagged NRAS was purified from HEK293T cells expressing Flag-tagged wt-TRAF7 or TRAF7 mutants by $\mathrm{Co}^{2+}$ metal affinity chromatography and detected by immunoblotting. E, A heatmap showing protein expression of RAS-related GTPases in HMCs expressing shGFP and shTRAF7. The scale shows Z-scored protein intensity values. $\mathrm{n}=3 \mathbf{F}$, Immunoblotting analysis of CDC42 and NRAS protein levels in HMCs expressing either shGFP or shTRAF7. G, A heatmap showing the change in mRNA expression of RAS-related GTPases in HMCs expressing shGFP and shTRAF7. The scale shows $\log 2$ (fold of change) of TPM. $\mathrm{n}=3$. H, Global protein stability (GPS) approach measuring NRAS, KRAS, HRAS, and CDC42 relative protein stability in HEK293T cells expressing shGFP or shTRAF7. Values are means $\pm \mathrm{SEM} ; \mathrm{n} \geq 4$. P values are from a two-sided Student's $\mathrm{t}$ test. I, Immunoblotting of the membrane and cytoplasmic fractions isolated from HMCs expressing shGFP or shTRAF7. $\boldsymbol{B}-\boldsymbol{D}, \mathrm{n}=3$. Numbers on the left are molecular masses in $\mathrm{kDa}$. 2xUb, two Ub; 1xUb, one Ub; EV, empty vector, WCL, whole-cell lysate. 
Figure 5. TRAF7 loss of function promotes tumorigenic transformation by inducing CDC42 activity. A, B, Serum-starved HMCs expressing shGFP or shTRAF7 were stimulated with MenCM, (Meningeal Cell Medium). The amount of GTP-bound CDC42 in human meningeal cells expressing shGFP or shTRAF7 was determined by pull down with RAC/CDC42 binding domain (PBD) conjugated to agarose beads. Data are shown as mean of GTP-bound CDC42 normalized to total CDC42 expression (A). Immunoblotting analysis using the indicated antibodies (B). C, D, Serum-starved HMCs expressing shGFP or shTRAF7 were stimulated with $2 \%$ serum. The amount of GTP-bound RAS in HMCs expressing the indicated vectors was determined by RAS GTPase Activation ELISA kit $(\boldsymbol{C})$. Immunoblotting analysis using the indicated antibodies $(\boldsymbol{D})$. E, Serum-starved HMCs expressing an empty vector (EV), wt-TRAF7, or TRAF7-E353* mutant were stimulated with $2 \%$ serum for the indicated time periods. The amount of GTP-bound RAS in HMCs expressing the indicated vectors was determined by RAS GTPase Activation ELISA kit. F, Serum-starved HMCs expressing an empty vector (EV) wt-TRAF7, TRAF7-T391I or TRAF7- N520S were stimulated with $2 \%$ serum for the indicated time periods. Immunoblotting analysis using the indicated antibodies. G, Serum-starved HMCs expressing empty vector (EV), wt-TRAF7, TRAF7-T391I or TRAF7- N520S were stimulated with MenCM for the indicated time periods. Immunoblotting analysis using the indicated antibodies. H, AI growth of HMCs expressing an empty vector (EV), CDC42-K163R or CDC42-G12V mutants. I, AI growth of HMCs expressing shRNAs targeting GFP or TRAF7 and treated with DMSO or $0.1 \mu \mathrm{M}$ FRAX597 for 7 days. Values are means \pm SEM; P values were detected by one-way ANOVA. n=3. J, AI growth of HMCs expressing an empty vector (EV) or ubiquitination-deficient mutant NRAS (UbDefNRAS) and shRNAs targeting GFP or TRAF7. K, AI growth of HMCs expressing an empty vector (EV) or NRAS-Q61K. $\boldsymbol{A}, \boldsymbol{C}, \boldsymbol{E}$, Values are means \pm SEM; P values were detected by two-way ANOVA. n=3. $\boldsymbol{H}, \boldsymbol{K}, \boldsymbol{J}$, Values are means \pm SEM. $\mathrm{n} \geq 3$. P values are from a two-sided Student's t test. 


\section{Figure 6. Cooperation of KLF4 and TRAF7 loss of function to promote tumorigenic}

transformation of human meningeal cells. A, KLF4 (probe ILMN_1779857) expression in adult meninges and meningioma subgroups (14). Boxplot showed the 25th, 50th and 75th percentile of expression values with whiskers expanding to 1.5 times the interquartile range of the data. $\mathrm{P}$ value is from a two-sided Student's t test. B, RT-qPCR analysis of KLF4 mRNA in HMCs expressing shGFP or shTRAF7. C, Immunoblotting analysis of KLF4 expression in HMCs expressing shGFP or shTRAF7. D, RT-qPCR analysis of TRAF7 and KLF4 expression in HMCs expressing shGFP or shTRAF7 treated with DMSO or Pimasertib (10 $\mu \mathrm{M}, 48$ hours). Values are means \pm SEM. $\mathrm{n} \geq 3$. P values are from a twoway ANOVA E, RT-qPCR analysis of KLF4 expression in HMCs expressing an empty vector (EV), CDC42-G12V, or NRAS-Q61K. F, Overlap of KLF4 target genes differentially expressed among HMCs overexpressing GFP or wt-KLF4, HMCs expressing shGFP or shTRAF7, and HBL52 expressing shGFP or shKLF4. KLF4 target genes were identified by the IPA upstream regulator analysis. G, H, I, RT-qPCR analysis of $C R A B P 2$ and $S E M A 3 F$ in HMCs expressing the indicated vectors. J, AI growth of HMCs expressing an empty vector (EV), CRABP2, or SEMA3F. K, Enrichment of the canonical IPA pathways in transcriptome analyses of HMCs overexpressing an empty vector (EV) or wt-KLF4; HMCs expressing shGFP or shTRAF7; HBL52 expressing shGFP or shKLF4; and KLF4/TRAF7-mutated meningiomas compared to wt-KLF4/mutant TRAF7 meningiomas. L, AI growth and the IPA upstream regulator analysis of HMCs expressing GFP, wt-KLF4, or KLF4-K409Q. M, N, O, AI growth of HMCs expressing the indicated constructs. gSCR, scramble gRNA, gKLF4, gRNA targeting KLF4. P, AI growth of $\mathrm{CH} 157-\mathrm{MN}$ cells expressing the indicated constructs. $\boldsymbol{B}, \boldsymbol{E}, \boldsymbol{G}, \boldsymbol{H}, \mathbf{I}, \boldsymbol{J}, \boldsymbol{L}-\boldsymbol{P}$, Values are means \pm SEM. $\mathrm{n} \geq 3$. P values are from a two-sided Student's t test. 

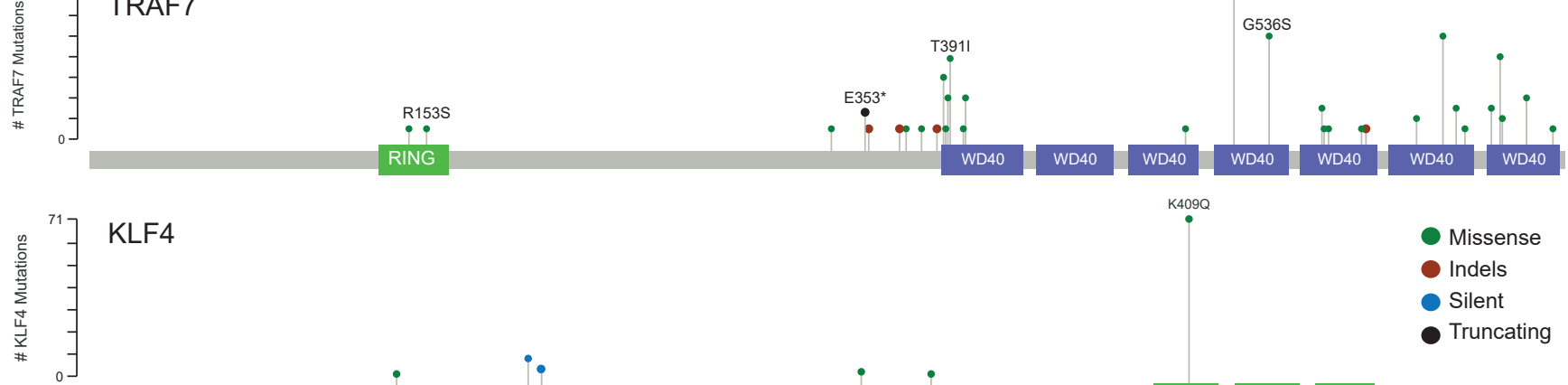

$409 \mathrm{Q}$

Missense

Indels

Silent

Truncating
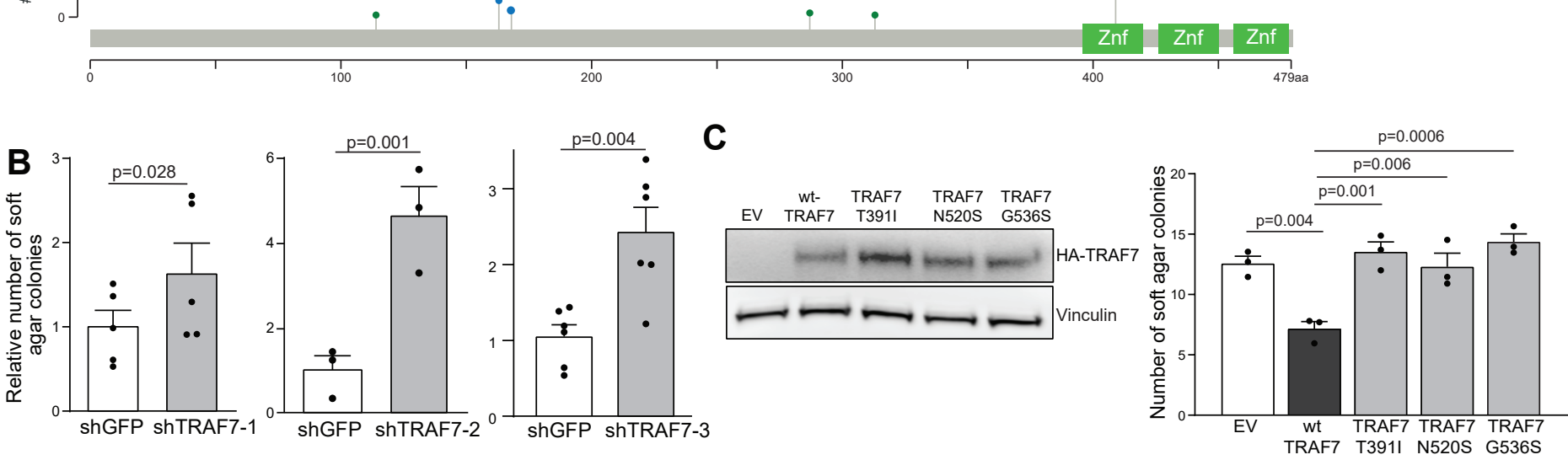

D

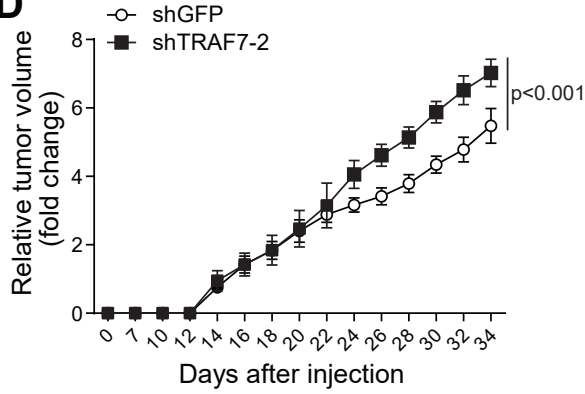

E

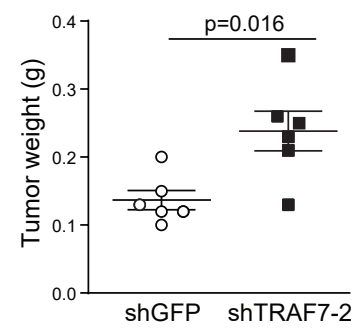

$\mathbf{F}$

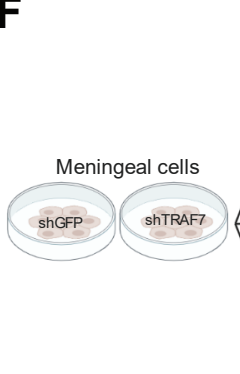

$\begin{array}{ll}\text { GC-peptide } & \text { LC-MS/MS } \\ \text { enrichment }\end{array}$

enrichment LC-MS/MS $\stackrel{\text { Analysis }}{3599} \underset{\text { ubiquitination }}{\longrightarrow} \stackrel{\text { FDR }<0.1}{\longrightarrow} 177$ genes $\longrightarrow$ sites quantified $\longrightarrow$ ubiquitinated LC-MS/MS $\begin{array}{lccc}\text { Analysis } & 2972 & \text { FDR }<0.05 & 709\end{array}$

$\longrightarrow \|_{m=2} \longrightarrow \begin{gathered}\text { proteins } \\ \text { quantified }\end{gathered} \longrightarrow \begin{gathered}\text { differentially } \\ \text { expressed } \\ \text { genes }\end{gathered}$

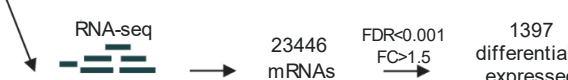

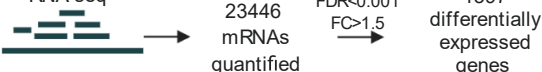

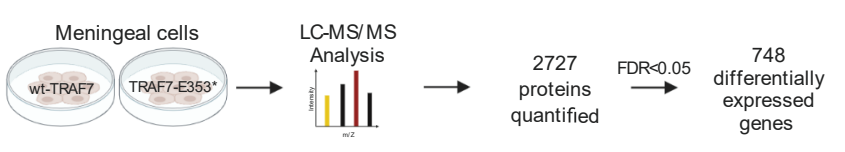

Pathway

Analysis

G

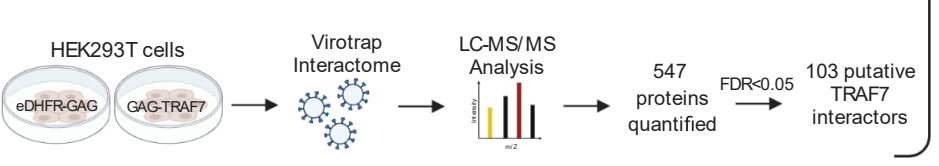

SIRTUIN_SIGNALING_PATHWAY

14_3_3_MEDIATED_SIGNALING CAVEOLAR_MEDIATED_ENDOCYTOSIS_SIGNALING

HEPATIC_FIBROSIS_HEPATIC_STELLATE_CELL_ACTIVATION REMODELING_OF_EPITHELIAL_ADHERENS_JUNCTIONS ROLE_OF_OSTEOBLASTS,_OSTEOCLASTS_AND_CHONDROCYTES_IN_RHEUMATOID_ARTHRITIS GLIOBLASTOMA_MULTIFORME_SIGNALING

GRANULOCYTE_ADHESION_AND_DIAPEDESIS

HEPATIC_FIBROSIS_SIGNALING_PATHWAY

ROLE_OF_MACROPHAGES,_FIBROBLASTS AND

ENDOTHHELIAL_CELLS_IN_RHEUMATOID_ARTHRITIS

CARDIAC_HYPERTROPHY_SIGNALING_(ENHANCED)

SUPERPATHWAY_OF_CHOLESTEROL_BIOSYNTHESIS

AXONAL GUIDANCE_SIGNALING

$\bigcirc$ INTEGRIN_Signaling

$\bigoplus_{10 N}$

$\bigcirc$

GERM_CELL_SERTOLI_CELL_JUNCTION_SIGNALING

ACtIN_nUCleAtION_BY_ARP_WASP_COMPLEX

ACTIN_NUCLEATION_BY_ARP_WASP_COMPLEX
CXCR4_SIGNALING RAC_SIGNALING ACTIN_CYTOSKELETON_SIGNALING NGF_SIGNALING RAC_SIGNALING ACTIN_CYTOS REGULATION_OF_EIF4_AND_P70S6K_SIGNALING MTOR_SIGNALING NRF2_MEDIATED_OXIDATIVE_STRESS_RESPONSE

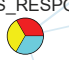

CELL_SIGNALING

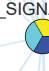

BAG2 SIGNALING PATHWAY

ALDOSTERONE_SIGNALING_IN_EPITHELIAL_CELLS

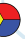

.

\section{EIF2_SIGNALING}

ay

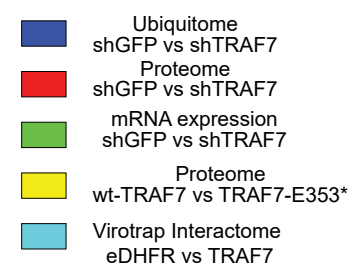
eDHFR vs TRAF7

\footnotetext{
S_ADENOSYL_L_METHIONINE_BIOSYNTHESIS ROLE_OF_PKR_IN_INTERFERON_INDUCTON_AND_ANTIVIRAL_RESPONSE
PROTEIN_UBIQUITINATION_PATHWAY MECHANISMS_OF_VIRAL_EXIT_FROM_HOST_CELLS

S_ADENOSYL_L_METHIONINE_BIOSYNTHESIS ROLE_OF_PKR_IN_INTERFERON_INDUCTON_AND_ANTIVIRAL_RESPONSE
PROTEIN_UBIQUITINATION_PATHWAY MECHANISMS_OF_VIRAL_EXIT_FROM_HOST_CELLS
} 
Figure 2

A

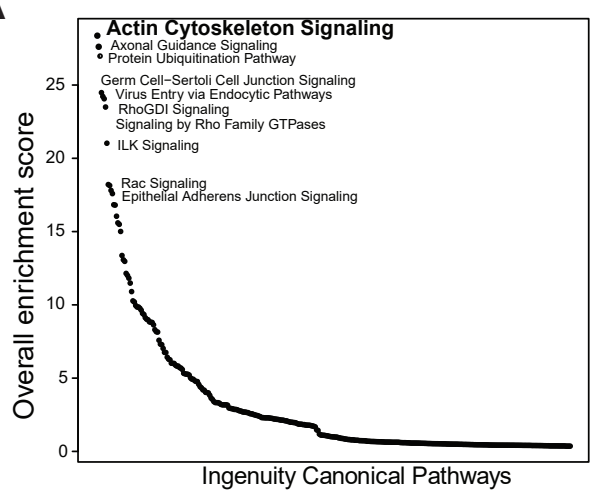

B

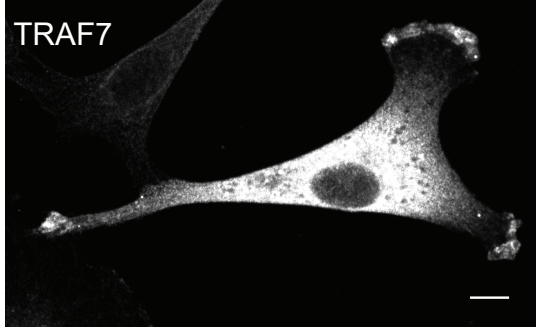

C
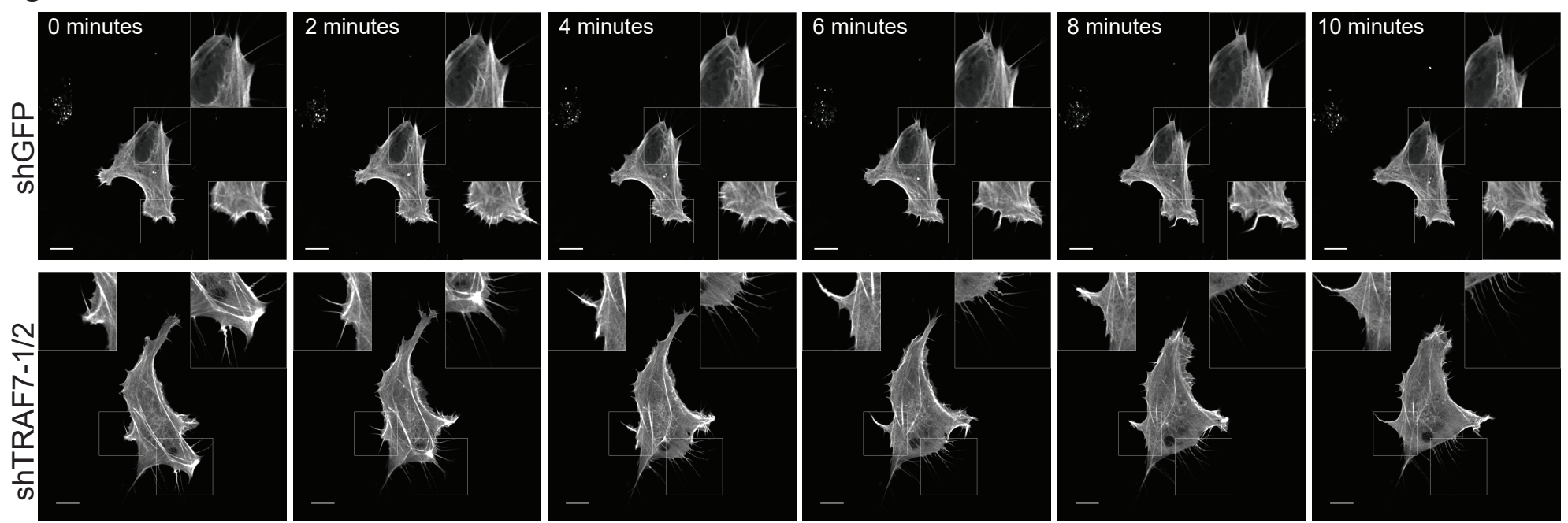

D
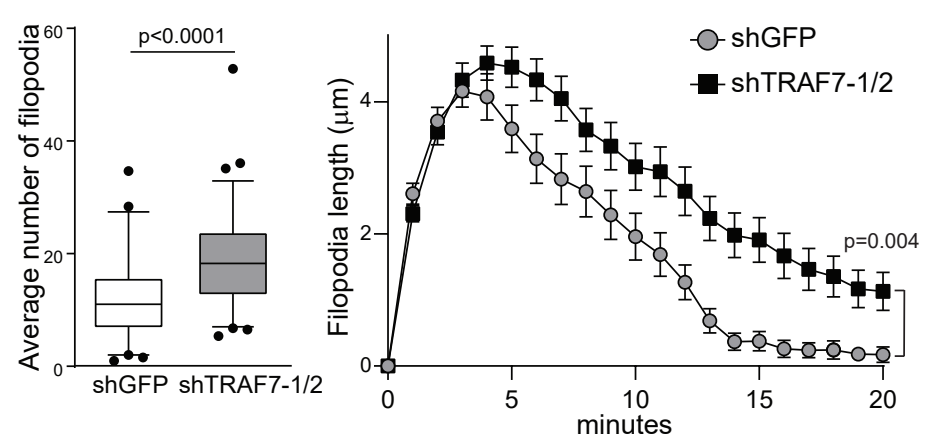

E
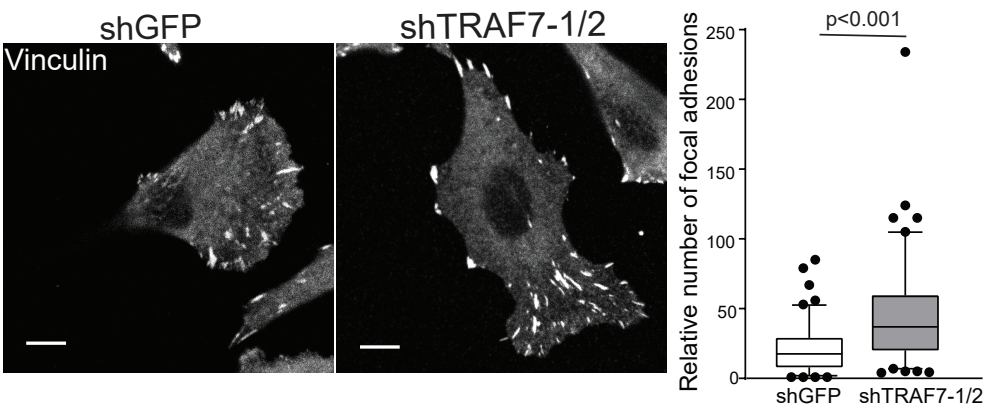

G
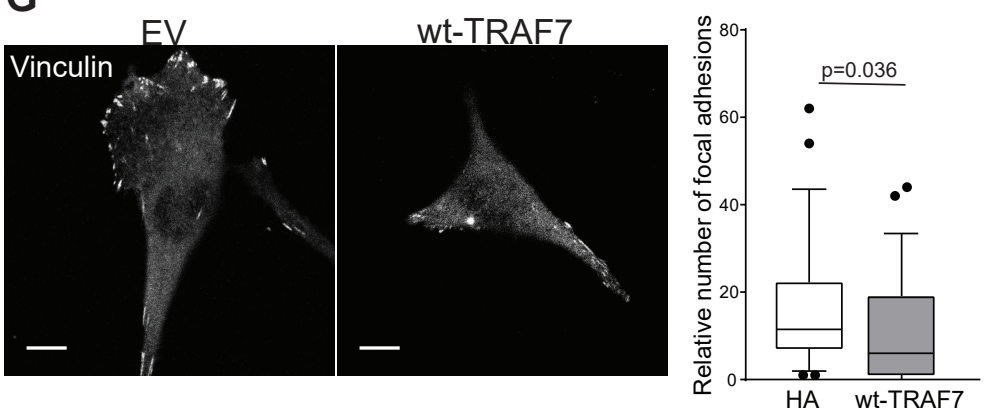

HA wt-TRAF7
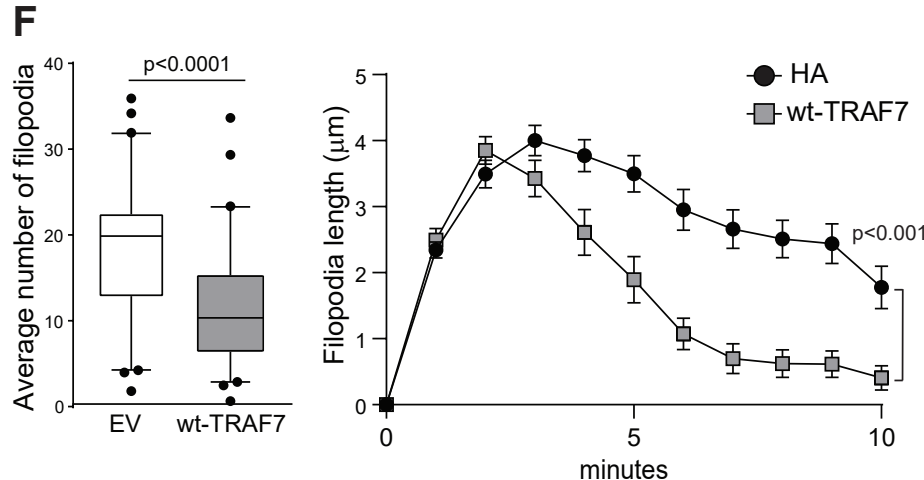
A

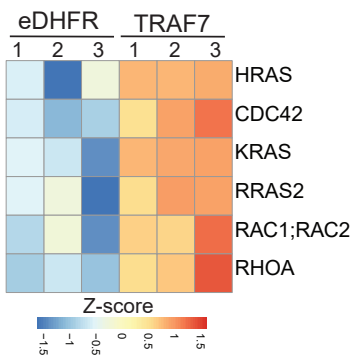

D

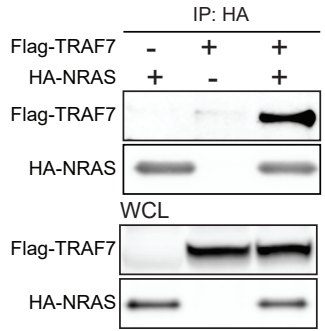

E

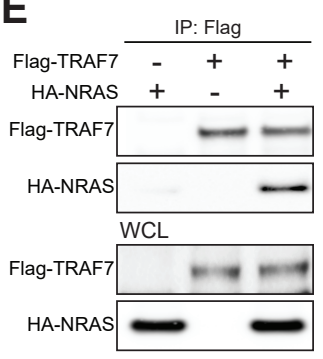

B

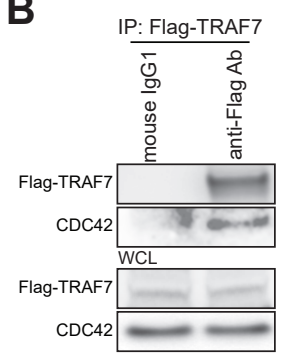

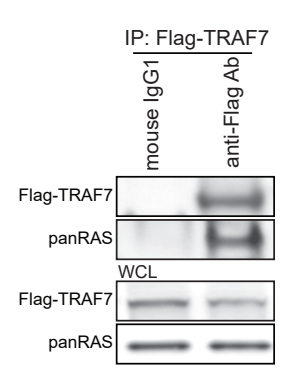
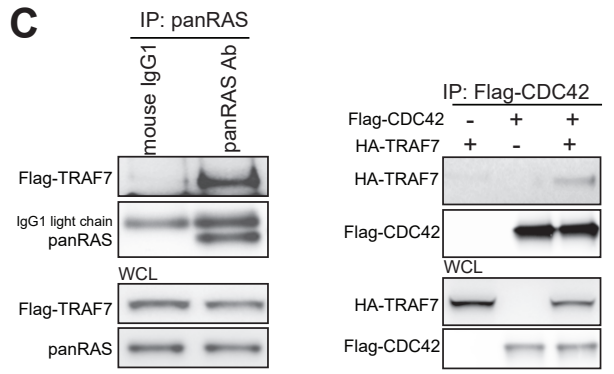

F
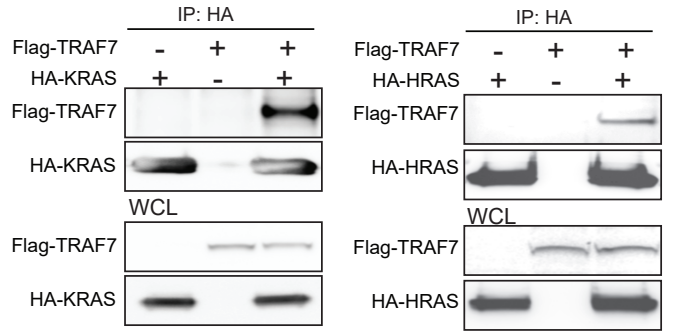

F
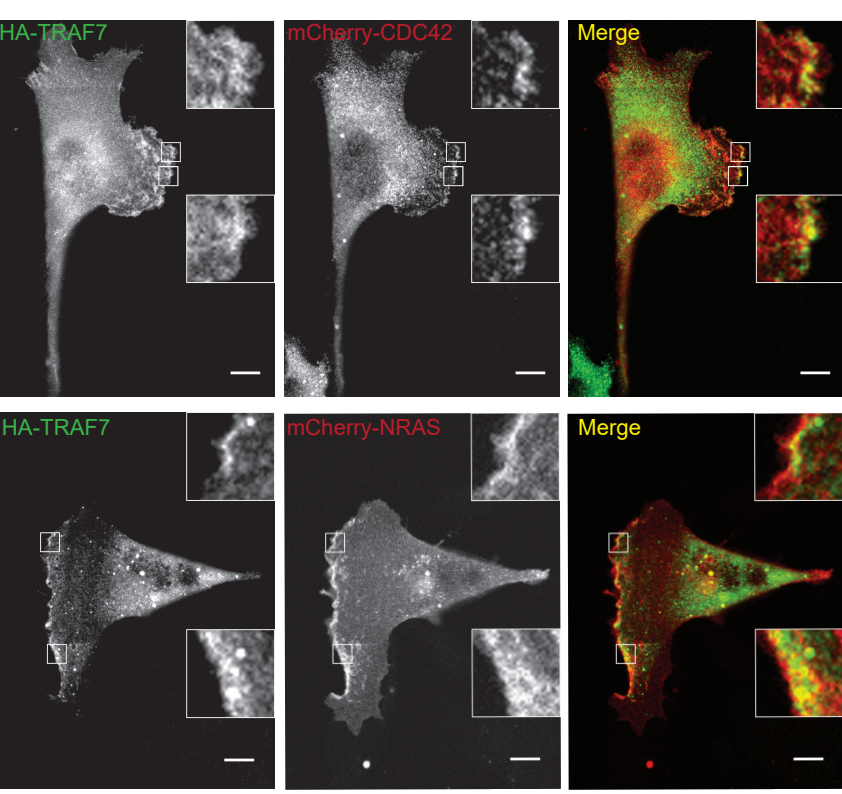

G

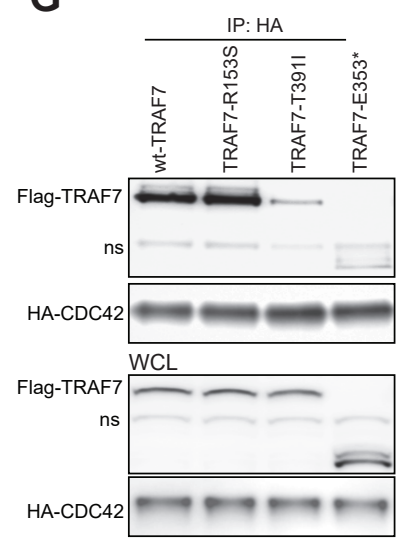

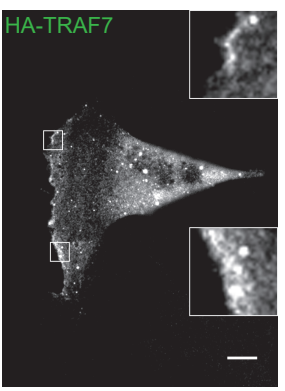

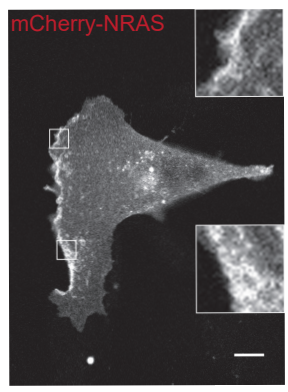

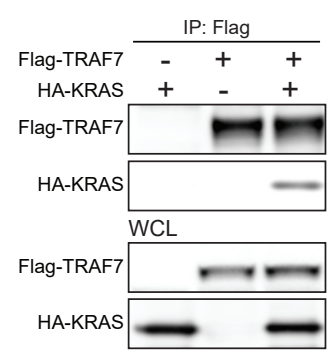

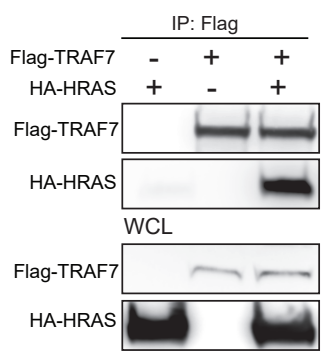

IP: HA
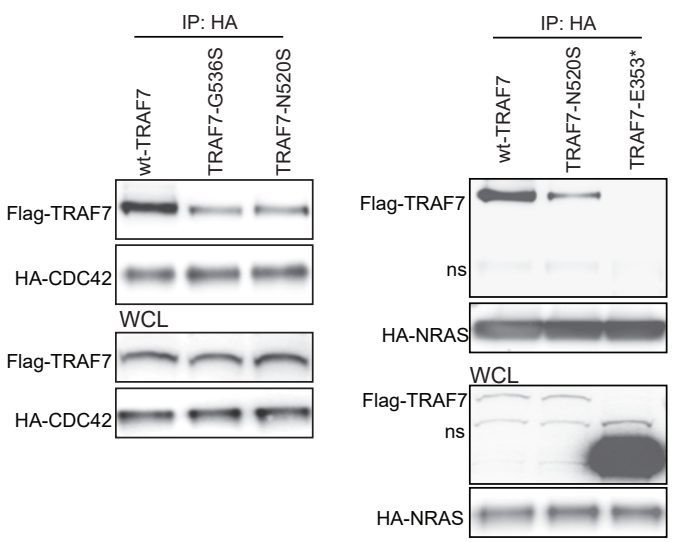
B

A

Ubiquitome

ShTRAF7-1/2/shGFP

$\begin{array}{lll}1 & 2 & 3 \\ \text { CDC42;RHOQ_163 }\end{array}$ CDC42_144 NRAS_128 RAC1;RAC3_184 RAC1_123 RAC1_133 RAC2_123 RHOA_135 RHOC;RHOA_118 RHOC;RHOA_119 RRAS2_177 $\log 2$ (fold of change) $\perp$ i $\circ$ ir
C

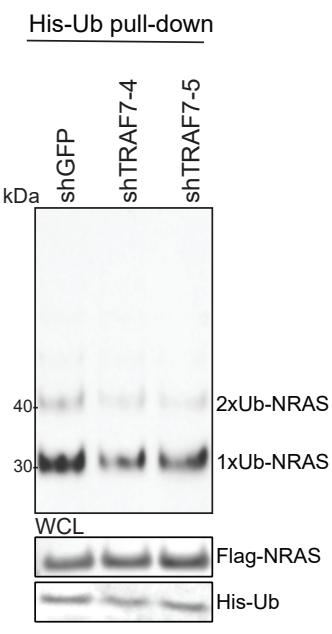

D

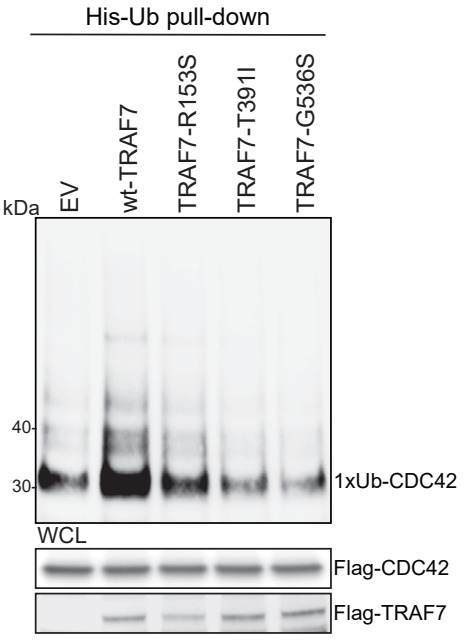

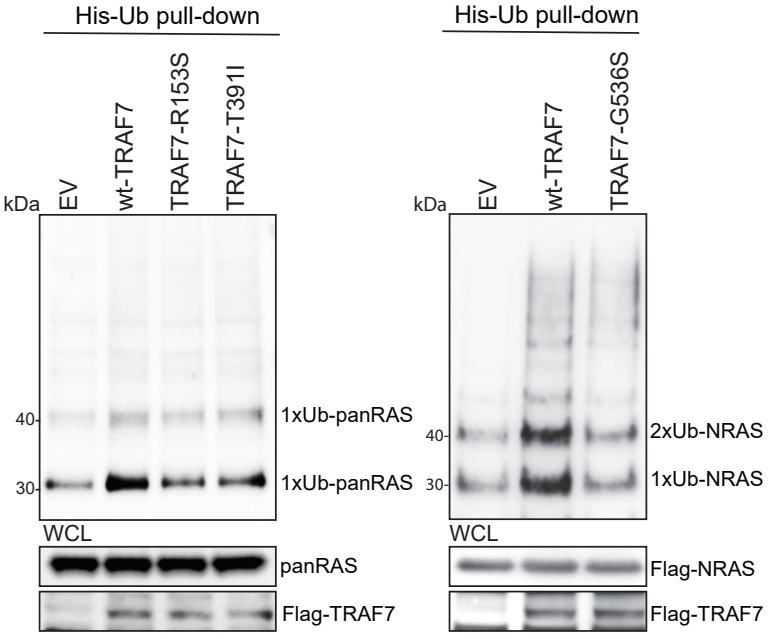

G

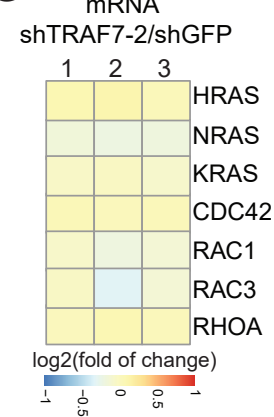

RAS RAS A
E

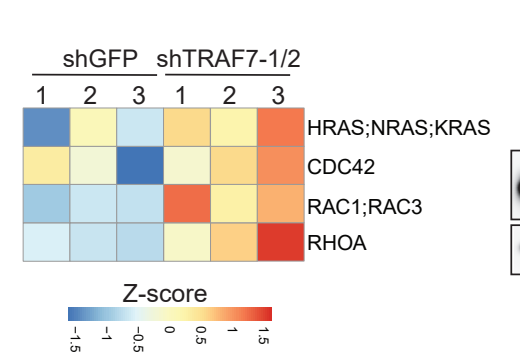

F

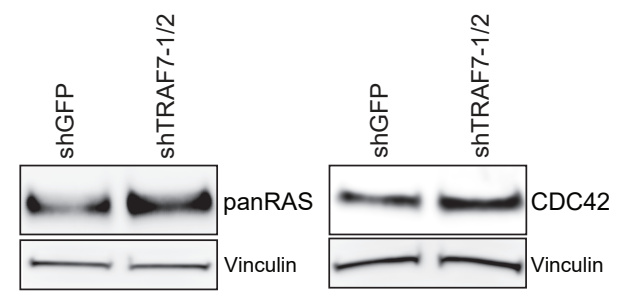

H

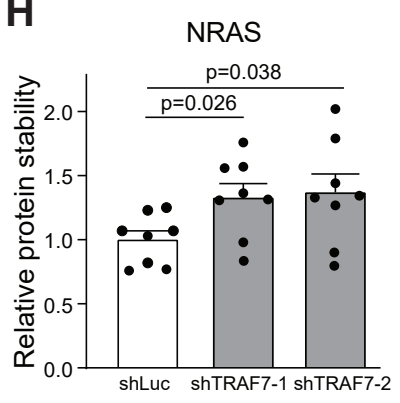

KRAS

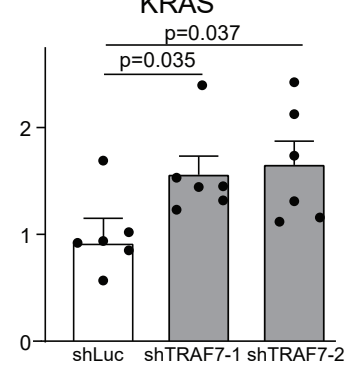

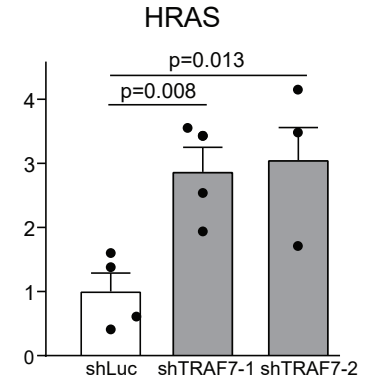

CDC42

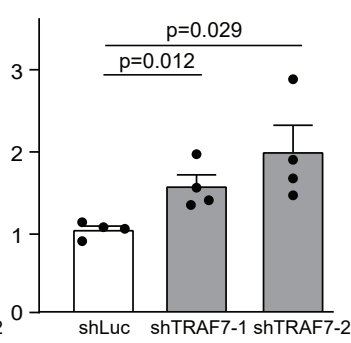

Cytoplasmic Membrane

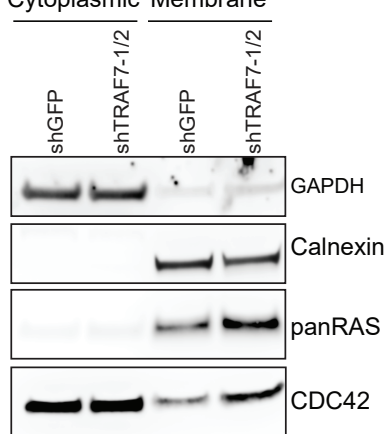


A

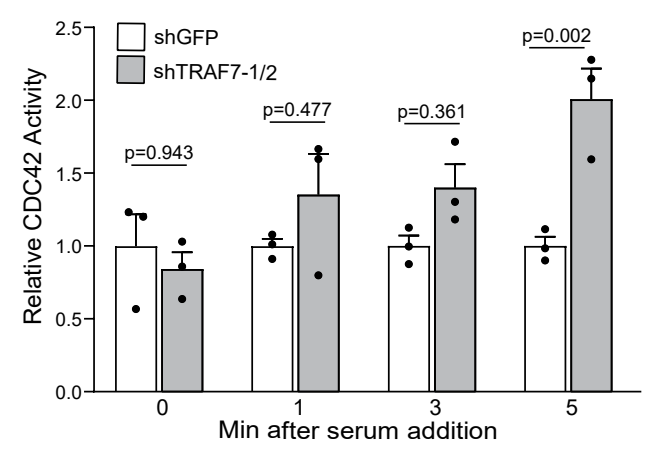

C

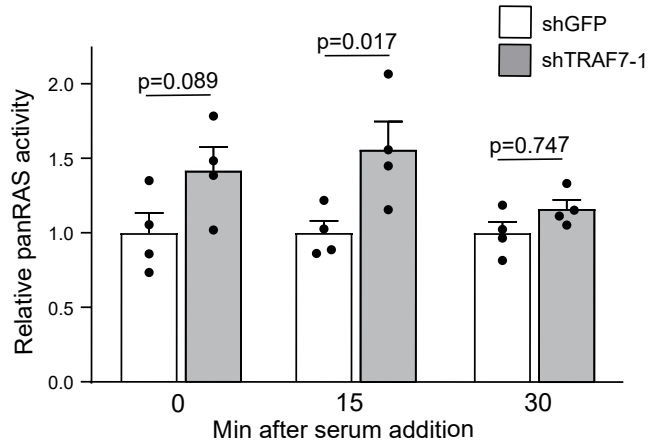

E

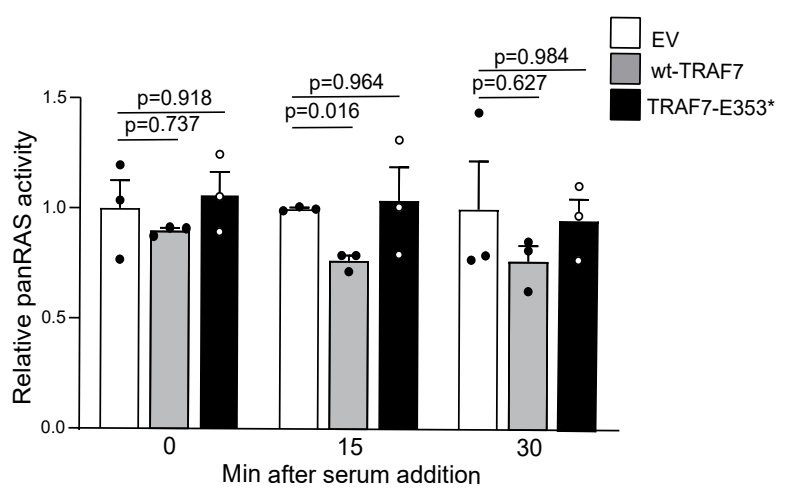

B

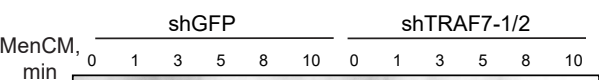

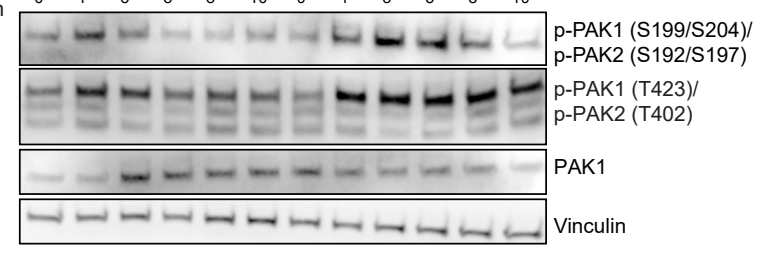

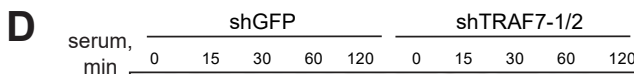

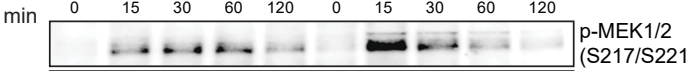

- - -

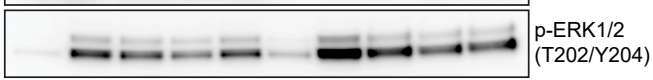

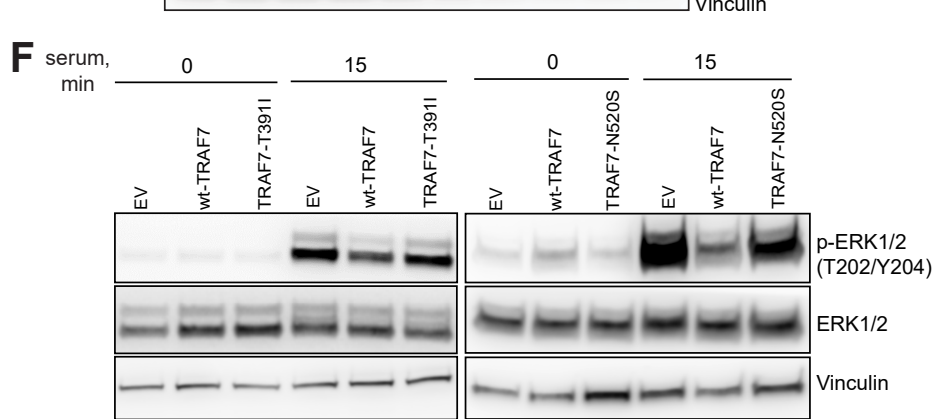

G

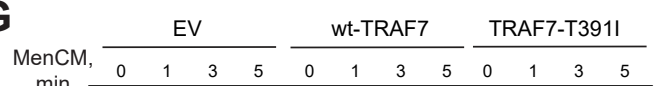

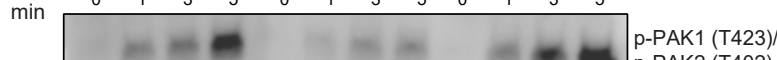

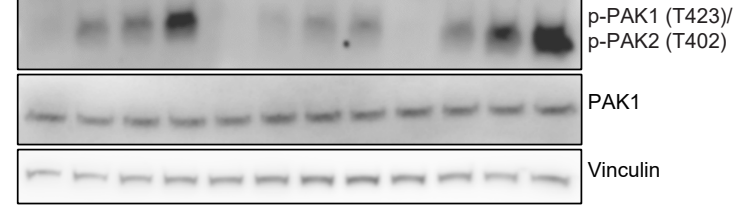

MenCM, $\frac{\text { EV }}{0 \quad 135} \frac{\text { wt-TRAF7 }}{0} \frac{\text { TRAF7-N520S }}{1}$

$\min$\begin{tabular}{|llllllllllll|}
0 & 1 & 3 & 5 & 0 & 1 & 3 & 5 & 0 & 1 & 3 & 5 \\
\hline & & & & & & & & & & & \\
\hline
\end{tabular}

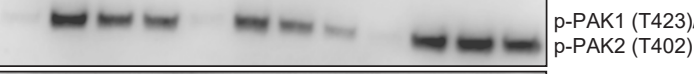

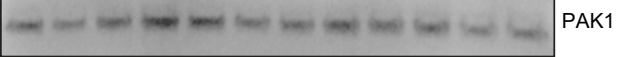
$\because \ldots+\cdots+\cdots m-n$ Vinculin
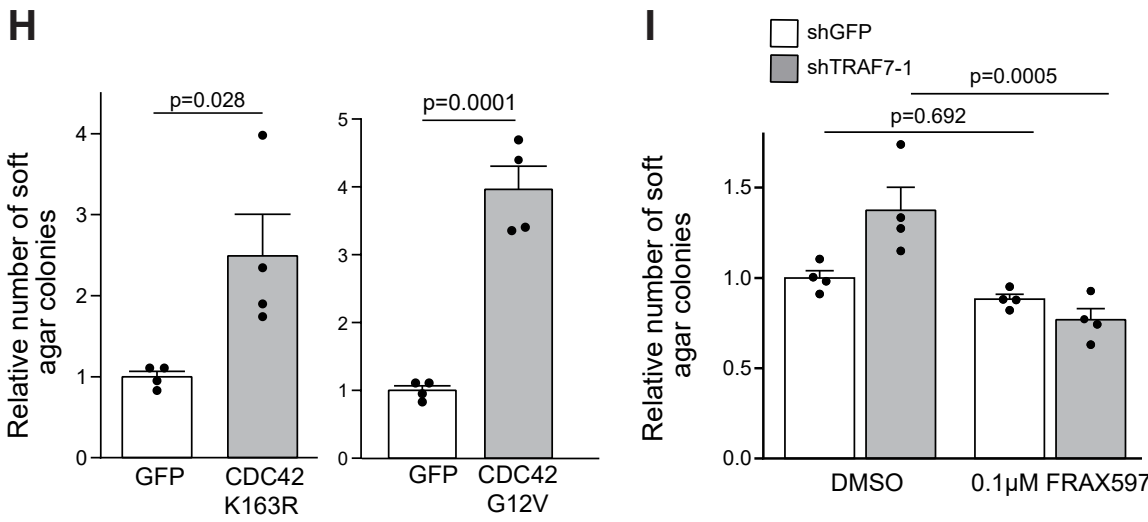

\section{$\square$ shGFP}

$\square$ shTRAF7-2
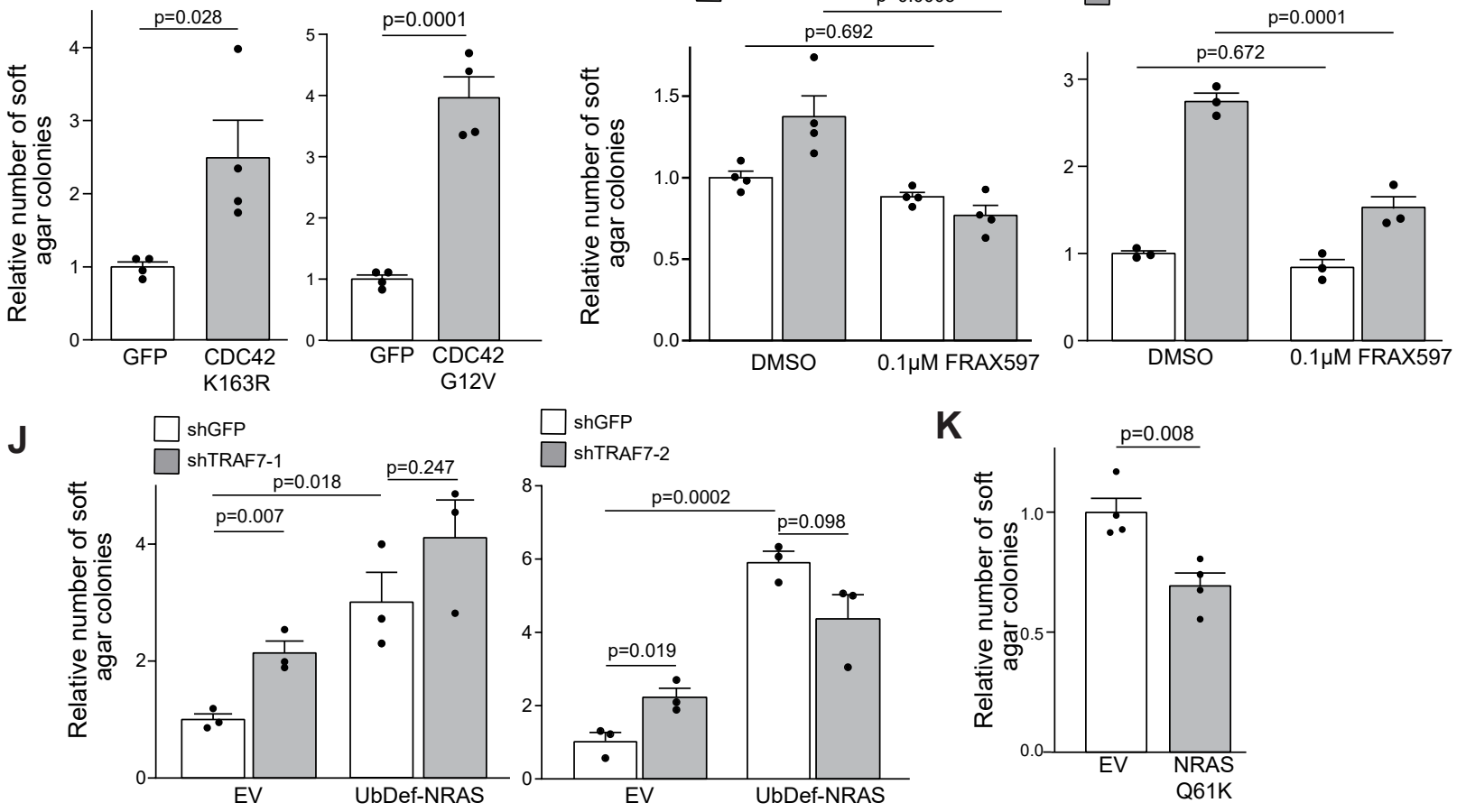

K

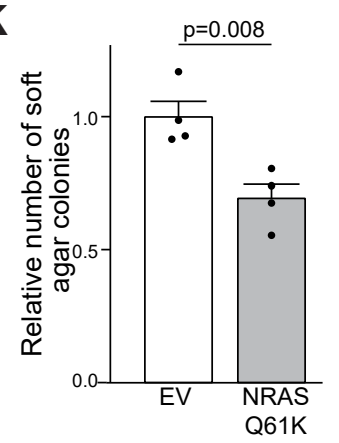


Figure 6
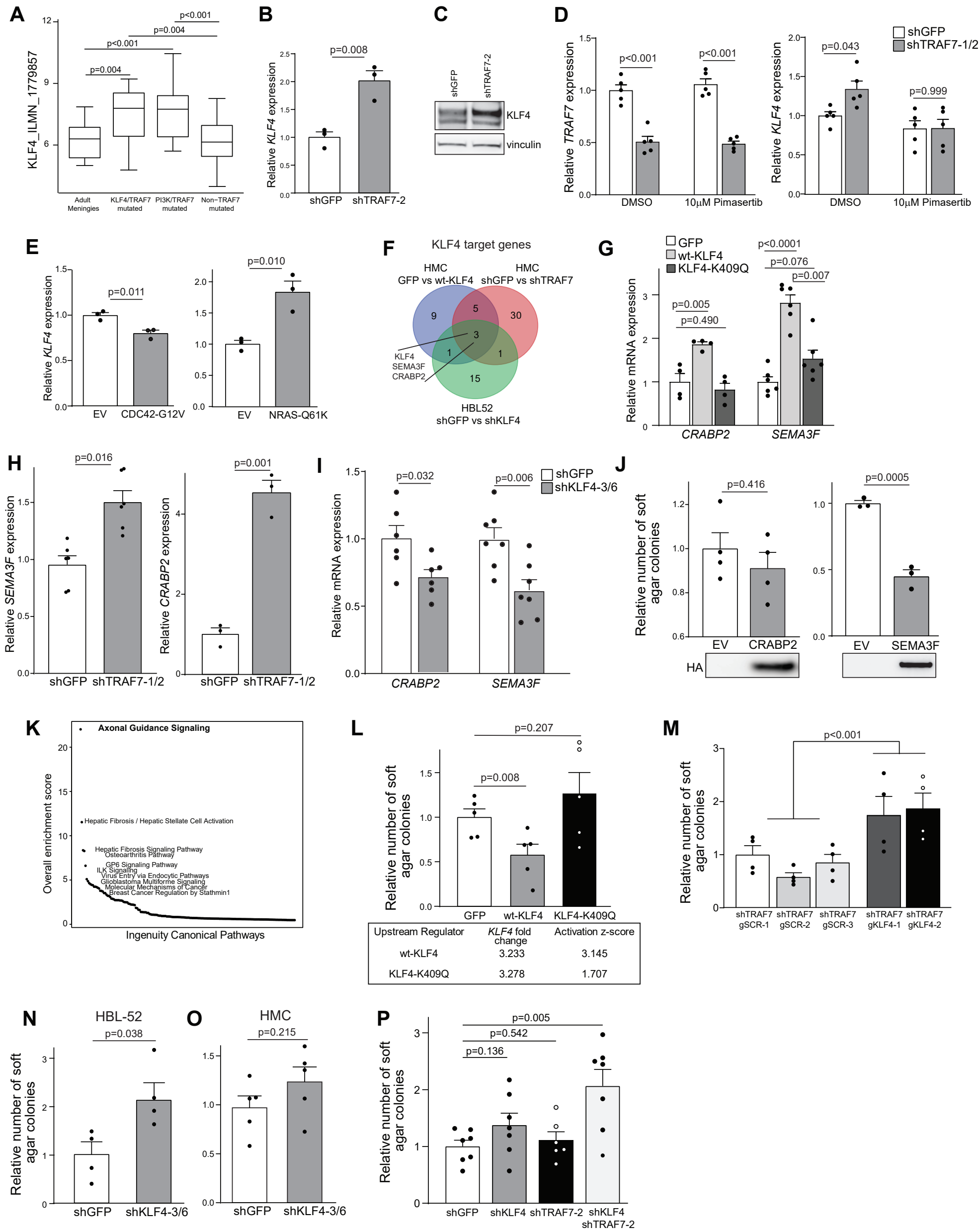


\section{Supplemental Material and Methods}

\section{Virotrap Screening}

The pMET7-GAG-TRAF7 construct was generated as described in (1). Virotrap experiments were performed as described in (2). Briefly, HEK293T cells were transfected pMET7-GAGTRAF7 or pMET7-GAG-eDHFR together with either untagged, or FLAG-tagged VSV-G using polyethylenimine (Sigma-Aldrich). 36 hours after transfection, particles were purified from the supernatant using MyOne paramagnetic streptavidin beads (ThermoFisher Scientific) preloaded with $2 \mu \mathrm{g}$ FLAG-Bio-M2 (Sigma-Aldrich). After washing with TRIS wash buffer (TWB: $20 \mathrm{mM}$ Tris- $\mathrm{HCl} \mathrm{pH}$ 7.5, $150 \mathrm{mM} \mathrm{NaCl}$ ), particles were eluted using the FLAG peptide (Sigma Aldrich) in TWB and processed by amphipols lysis and precipitation under acidic conditions. After resuspension in triethylammonium bicarbonate (TEAB) buffer and boiling, proteins were overnight digested with sequencing-grade trypsin (Promega). After acidic precipitation of partially or undigested proteins by amphipols and acidification, the sample was analyzed by MS.

Of each sample, $5.0 \mu \mathrm{L}$ was introduced into an LC-MS/MS system through an Ultimate 3000 RSLC nano LC (Thermo Fisher Scientific, Bremen, Germany) in-line connected to a Q Exactive HF mass spectrometer (Thermo Fisher Scientific). The sample mixture was first loaded with loading solvent $(0.1 \%$ TFA in water/acetonitrile, $98 / 2(\mathrm{v} / \mathrm{v}))$ on a trapping column (made in-house, $100 \mu \mathrm{m}$ internal diameter (I.D.) $\times 20 \mathrm{~mm}, 5 \mu \mathrm{m}$ beads C18 Reprosil-HD, Dr. Maisch, Ammerbuch-Entringen, Germany). After flushing from the trapping column, the sample was loaded on an analytical column (made in-house, $75 \mu \mathrm{m}$ I.D. $\times 200 \mathrm{~mm}, 1.9 \mu \mathrm{m}$ beads C18 Reprosil-HD, Dr. Maisch) packed in the needle (pulled in-house). Peptides were eluted with a non-linear 60 min gradient from $98 \%$ solvent A ( $0.1 \%$ formic acid in MQ) reaching $55 \%$ solvent $\mathrm{B}(0.1 \%$ formic acid in water/acetonitrile, $20 / 80(\mathrm{v} / \mathrm{v}))$ in $50 \mathrm{~min}$ followed by an increase to $97 \%$ solvent $B$ in $10 \mathrm{~min}$ at a flow rate of $250 \mathrm{~nL} / \mathrm{min}$. This was followed by 
a 10 min wash reaching $99 \%$ solvent $\mathrm{B}$ and re-equilibration with solvent A. Column temperature was kept constant at $50^{\circ} \mathrm{C}$ (Butterfly nano-LC column oven, Phoenix S\&T).

The mass spectrometer was operated in data-dependent, positive ionization mode, automatically switching between MS and MS/MS acquisition for the 16 most abundant peaks in a given MS spectrum. The source voltage was set to $2.5 \mathrm{kV}$ and the capillary temperature was $250^{\circ} \mathrm{C}$. One MS1 scan (m/z 375-1500, AGC target 3E6 ions, maximum ion injection time of $80 \mathrm{~ms}$ ) acquired at a resolution of 60,000 (at $200 \mathrm{~m} / \mathrm{z}$ ) was followed by up to 16 tandem MS scans (resolution 15,000 at $200 \mathrm{~m} / \mathrm{z}$ ) of the most intense ions fulfilling predefined selection criteria (AGC target 1E5 ions, maximum ion injection time of $80 \mathrm{~ms}$, isolation window of 1.5 $\mathrm{m} / \mathrm{z}$, fixed first mass of $145 \mathrm{~m} / \mathrm{z}$, spectrum data type: profile, underfill ratio $2 \%$, intensity threshold 1.3E4, exclusion of unassigned, singly and $>7$ charged precursors, peptide match preferred, exclude isotopes on, dynamic exclusion time of $12 \mathrm{~s}$ ). The HCD collision energy was set to $30 \%$ Normalized Collision Energy and the polydimethylcyclosiloxane background ion at 445.12002 Da was used for internal calibration.

The RAW Virotrap MS data were searched using MaxQuant (version 1.5.8.3) against the human Uniprot/SwissProt database, complemented with GAG, VSVG, and eDHFR protein sequences. The searches were performed with $4.5 \mathrm{ppm}$ tolerance on precursor and $20 \mathrm{ppm}$ tolerance on fragment mass, and with trypsin/P settings allowing up to two missed cleavages. Methionine oxidation and N-terminal acetylation formation were set as variable modifications. Minimum peptide length was set to 7 , and maximum peptide mass was 4600 Da. PSM FDR and protein FDR were set to 0.01 . Minimum peptides and minimum razor + unique peptides were set to 1 . The searches were performed together with the eDHFR control samples to enable matching of MS spectra between runs. Contaminants and identifications against the reverse database hits were removed using the Perseus software (version 1.5.5.3).

\section{Proteome and Ubiquitome analyses}


100 million cells were washed twice with PBS and lysed in $10 \mathrm{ml}$ urea lysis buffer containing 9 $\mathrm{M}$ urea and $20 \mathrm{mM}$ HEPES $\mathrm{pH}$ 8.0. Samples were sonicated with 3 pulses of $15 \mathrm{~s}$ at an amplitude of $20 \%$ using a $3 \mathrm{~mm}$ probe, with incubation on ice for 1 minute between pulses followed by centrifugation for 15 minutes at $15,000 \mathrm{x} g$ at room temperature. $10 \mathrm{mg}$ of total protein per sample was used for further analysis. Proteins in each sample were reduced by incubating with $4.5 \mathrm{mM}$ DTT for 30 minutes at $55^{\circ} \mathrm{C}$. Alkylation of the proteins was done by addition of $10 \mathrm{mM}$ chloroacetamide for 15 minutes at room temperature. The samples were diluted with $20 \mathrm{mM}$ HEPES pH 8.0 to a urea concentration of $4 \mathrm{M}$ and the proteins were digested with lysyl endopeptidase (Wako, 1/100, w/w) for 4 hours at $37^{\circ} \mathrm{C}$. All samples were further diluted with $20 \mathrm{mM}$ HEPES $\mathrm{pH} 8.0$ to a final urea concentration of $2 \mathrm{M}$ and proteins were digested with trypsin (Promega, 1/100, w/w) overnight at $37^{\circ} \mathrm{C}$. The resulting peptide mixtures was purified on Sep-Pak C18 cartridges (Waters), lyophilized for two days and redissolved in the immunoprecipitation buffer of the PTMScan ${ }^{\circledR}$ Ubiquitin Remnant Motif (K- $\varepsilon-$ GG) Kit (Cell Signaling Technology). Aliquots corresponding to $200 \mu \mathrm{g}$ of digested protein material were taken for shotgun proteomics analysis. Immunocapture of GG-modified peptides was then performed using the PTMScan Kit according to the manufacturer's instructions. Peptides were incubated with the antibody-bead slurry for 2 hours on a rotator at $4^{\circ} \mathrm{C}$. GGmodified peptides were later eluted in $10 \mu 10.15 \%$ TFA and desalted on reversed phase C18 OMIX tips (Agilent). Purified GG-modified peptides were dried under vacuum in MS vials and stored at $-20^{\circ} \mathrm{C}$ until LC-MS/MS analysis.

For ubiquitome analysis, purified GG-modified peptides were re-dissolved in $20 \mu$ loading solvent A $(0.1 \%$ TFA in water/ACN $(98: 2, \mathrm{v} / \mathrm{v}))$ of which $15 \mu \mathrm{l}$ was injected for LC-MS/MS analysis. For proteome analysis, purified peptides for shotgun proteomics were re-dissolved in 
20 ul solvent A, peptide concentration as determined on a Lunatic spectrophotometer and approximately $3 \mu \mathrm{g}$ of peptides was injected for LC-MS/MS analysis. Both ubiquitome and proteome samples were analyzed on a Ultimate 3000 RSLCnano system in-line connected to a Q Exactive HF mass spectrometer (Thermo Scientific). The sample mixture was first loaded on a trapping column (made in-house, $100 \mu \mathrm{m}$ I.D. x $20 \mathrm{~mm}$ length, $5 \mu \mathrm{m}$ beads C18 ReprosilHD, Dr. Maisch). After flushing from the trapping column, the sample was loaded on a reversephase column (made in-house, $75 \mu \mathrm{m}$ I.D. x $200 \mathrm{~mm}$ length, $1.9 \mu \mathrm{m}$ beads C18 Reprosil-HD, Dr. Maisch). Purified GG-modified peptides and peptides for shotgun analysis were loaded with loading solvent A and were separated with a non-linear 116 min or 140 min gradient, respectively, from $2 \%$ to $56 \%$ solvent $\mathrm{B}(0.1 \%$ formic acid in water/ACN $20: 80(\mathrm{v} / \mathrm{v}))$ at a flow rate of $250 \mathrm{nl} / \mathrm{min}$ and at a constant temperature of $50^{\circ} \mathrm{C}$. Following a $10 \mathrm{~min}$ wash reaching $97 \%$ solvent $\mathrm{B}$, the column was re-equilibrated with solvent $\mathrm{A}(0.1 \%$ formic acid in water). For proteome analysis, the mass spectrometer was operated in data-dependent, positive ionization mode, automatically switching between MS and MS/MS acquisition for the 16 most abundant peaks in a given MS spectrum. One MS1 scan (m/z 375-1500, AGC target 3 E6 ions, maximum ion injection time of $60 \mathrm{~ms}$ ), acquired at a resolution of 60,000 (at $200 \mathrm{~m} / \mathrm{z}$ ) was followed by up to 16 tandem MS scans, acquired at a resolution of 15,000 (at $200 \mathrm{~m} / \mathrm{z}$ ) of the most intense ions fulfilling predefined selection criteria including an AGC target set at 1E5 ions, a maximum ion injection time of $80 \mathrm{~ms}$, an isolation window of $1.5 \mathrm{~m} / \mathrm{z}$, an intensity threshold of $1.3 \mathrm{E} 4$, exclusion of unassigned and singly charged precursors and a dynamic exclusion time of $12 \mathrm{~s}$. The normalized collision energy was set to $28 \%$ and the polydimethylcyclosiloxane background ion at 445.12003 Da was used for internal calibration. The ubiquitinome analysis was carried out in a similar way but with a maximum injection time of $120 \mathrm{~ms}$ and an intensity threshold of 8.3E3 as most important differences for peptide MS/MS selection. QCloud was used to control instrument longitudinal performance during the project. 
Data analysis was performed with MaxQuant (version 1.6.1.0) using the Andromeda search engine with default search settings including a false discovery rate set at $1 \%$ on the PSM, peptide and protein level. Three different searches were performed to analyze the spectra from the GG-enriched samples and the shotgun samples separately. In all searches spectra were interrogated against the human proteins in the Uniprot/Swiss-Prot database.

The mass tolerance for precursor and fragment ions was set to 4.5 and $20 \mathrm{ppm}$, respectively, during the main search. Enzyme specificity was set as C-terminal to arginine and lysine, also allowing cleavage at proline bonds with a maximum of three missed cleavages. Variable modifications were set to oxidation of methionine residues, acetylation of protein N-termini and GG modification of lysine residues, while carbamidomethylation of cysteine residues was set as fixed modification. The minimum score for modified peptides was set to 30 . Matching between runs was enabled and only proteins with at least one unique or razor peptide were retained. Proteins were quantified by the MaxLFQ algorithm integrated in the MaxQuant software. A minimum ratio count of two unique or razor peptides was required for quantification. Further data analysis was performed with the Perseus software after loading the proteinGroups and GG (K) sites tables from MaxQuant. Reverse database hits were removed as well as potential contaminants. The data of the protein groups table and was $\log 2$ transformed, proteins with less than three valid values in at least one group were removed and missing values were imputed from a normal distribution around the detection limit. The same steps were performed on the GG modified sites after expanding the sites table. The mass spectrometry proteomics data have been deposited to the ProteomeXchange Consortium via the PRIDE partner repository.

\section{Analysis of protein expression, activity, and interactions}

Ubiquitinated proteins were purified as described previously in (3). Briefly, cells were lysed in the IP lysis buffer containing EDTA-free protease inhibitor cocktail (Roche). Cell lysates were 
mixed with His-buffer A (PBS, $\mathrm{pH}$ 8.0, $6 \mathrm{M}$ guanidinium-HCl, 0.1\% NP-40 and $1 \mathrm{mM} \beta$-ME) and added to TALON $\mathrm{Co}^{2+}$ coated beads (Clontech) for 3 hours. The beads were washed one time with His-buffer A, three times with His-buffer B (PBS, pH 8.0, 0.1\% NP-40, 5\% glycerol, $20 \mathrm{mM}$ imidazole), and then proteins were eluted from the beads with $500 \mathrm{mM}$ imidazole solution for 30 minutes at room temperature.

Subcellular fractionation was performed according to manufacturer's protocol (ThermoFisher). Briefly, cells were scraped with ice-cold Cytoplasmic Extraction Buffer containing protease inhibitors, incubated at $4^{\circ} \mathrm{C}$ for 10 minutes and centrifuged at $500 \mathrm{~g}$ for 5 minutes at $4^{\circ} \mathrm{C}$. The supernatant containing cytoplasmic fraction was collected. The pellets were incubated with icecold Membrane Extraction Buffer containing protease inhibitors at $4^{\circ} \mathrm{C}$ for 10 minutes. Samples were cleared by centrifuging at $3000 \mathrm{~g}$ for 5 minutes $4^{\circ} \mathrm{C}$, and the supernatant containing membrane fraction was collected. Equal volumes of extracted protein lysates were loaded and separated by SDS-PAGE and detected by immunoblotting.

For immunoblotting, equivalent amounts of cell lysates were separated on 4-12\% gradient gels (Invitrogen), transferred to nitrocellulose membranes, and incubated with the indicated antibodies. The signal was visualized by chemiluminescence (Amersham Pharmacia Biotech) using an automated digital developer.

RAS activity was assessed using the RAS activation ELISA assay Kit (EMD Millipore). Cells were washed twice with ice-cold TBS and scraped on ice in Lysis Buffer containing protease and phosphatase inhibitor cocktails (Roche) and centrifugated for 10 minutes $16,000 \mathrm{~g}$ at $4^{\circ} \mathrm{C}$. Glutathione-coated 96-well plate was incubated with GST-tagged RAF1-RAS Binding Domain (RBD). After incubation with $10 \mu \mathrm{g}$ of protein lysate, the plate was washed and then incubated with anti-RAS antibody (Millipore, Clone RAS10), followed by secondary HRP-conjugated antibody. The signal was detected after addition of Chemiluminescent Substrate and recorded using Victor X3 Multi-label Plate Reader (PerkinElmer). 
CDC42 activation assay was performed according to the manufacturer's protocol (Cytoskeleton, Inc). Cell were washed with cold PBS and lysed in Cell Lysis Buffer supplemented with Protease Inhibitor Cocktail (Cytoskeleton, Inc) and centrifugated for 2 min $10,000 \mathrm{~g}$ at $4^{\circ} \mathrm{C} .400 \mu \mathrm{g}$ of cell lysates was mixed with GST-tagged PAK-PBD agarose beads (Cytoskeleton, Inc) and incubated for 60 minutes at $4{ }^{\circ} \mathrm{C}$. The beads were washed with Wash Buffer once and eluted by boiling for 5 minutes in 2x Laemmli sample buffer. Eluted proteins were separated by SDS-PAGE and detected by immunoblotting.

\section{Live imaging}

To perform live imaging, cells were plated on Nunc ${ }^{\text {TM }}$ Glass Bottom Dishes $27 \mathrm{~mm}$ (Thermo Fisher) and transfected with tdTomato-Lifeact-7 using Lipfectamine 3000 (Thermo Fisher). For image acquisition, we used a Nikon TiE inverted A1R (+ HD resonant scanning upgrade) microscope with a 40x Oil objective (NA 1,3) equipped with an Okolab incubator at $37^{\circ} \mathrm{C}$ and $5 \% \mathrm{CO}_{2}$. The setup was controlled by NIS-Elements (NIS 5.11.01 build 1368a, Nikon Instruments Europe B.V.). The length, size, and the number of protrusions per cell per time point were analyzed using a so-called general analysis protocol (GA2) in NIS-elements (NIS 4.50.00 build 1117, Nikon Instruments Europe B.V.) using standard image processing procedures. In short, the images were deconvolved, and transfected cells were identified using an intensity threshold. Objects touching image borders and not fitting the expected cell size, like clusters or debris, were excluded. Consequently, images were segmented to identify the complete cells with the protrusions, the rough cell outline, and the protrusions separately. Complete cells were identified using a threshold and by closing holes in the objects. The rough outline of the cells was identified using a combination of smoothing, erosion, and dilation steps together with size exclusion filters to remove isolated parts of protrusions. The protrusions were selected by the differential of the rough outline and the complete cells. The intersection of the 
protrusions and a dilated mask of the rough cell outline yielded the number of protrusions per cell.

\section{Bioinformatic analyses}

mRNA expression levels were estimated by RSEM (4) using GENCODE reference annotation of the human genome (GRCh38), version 22 (Ensembl 79). Differential expression analysis was performed using the DESeq2 $\mathrm{R}$ package. The significance was determined by FDR $<0.01$ (Figure 1) or FDR<0.05 (Figure 6) and fold change >1.5 (GEO Series GSE156211).

The expression microarray data of meningiomas were previously published in (5) (GEO Series GSE84263). The KLF4 expression probe was compared between mutation groups using twotailed Student's t-test. We also used The $\mathrm{R}$ package Limma was used to identify the differentially expressed genes. FDR $<0.05$ and a fold change $>1.5$ were used as cutoff to determine differentially expressed genes. Differentially expressed genes were used as input for the IPA core pathway analysis.

To rank the enrichment of the pathways among the multi-omics dataset, we created the overall enrichment score. For each pathway, the overall enrichment score was calculated by: $\frac{\operatorname{Sum}(-\log 10(\mathrm{P} \text { value }))}{1 / \mathrm{n}}$, where $\operatorname{sum}(-\log 10(\mathrm{P}$ value $))$ is the total sum of the $-\log 10(\mathrm{P}$ value $)$ from IPA of all datasets used, $\mathrm{n}$ is the number of datasets of which the pathway is significantly enriched. The $\mathrm{P}$ value $<0.01$ was used to determine the significance of the pathway enrichment. If the pathway was not significantly enriched in a dataset, the $-\log 10$ (P value) was set to 0 .

For identification of TRAF7 mutations, paired-end reads have been aligned against the human reference genome hg19 using BWA (6). The SAMtools (7) was used to convert and sort the sam files output from BWA to bam files. The mpileup command from SAMtools further converted the sorted bam files to the pileup format as input for the germline SNPs calling using Varscan2 (8). SnpEff (9) was used to annotate the SNPs and predict the functional consequence of these SNPs. 


\section{References}

1. Eyckerman S, Titeca K, Van Quickelberghe E, Cloots E, Verhee A, Samyn N, et al. Trapping mammalian protein complexes in viral particles. Nat Commun 2016;7:11416

2. Titeca K, Van Quickelberghe E, Samyn N, De Sutter D, Verhee A, Gevaert K, et al. Analyzing trapped protein complexes by Virotrap and SFINX. Nat Protoc 2017;12:881-98

3. Simicek M, Lievens S, Laga M, Guzenko D, Aushev VN, Kalev P, et al. The deubiquitylase USP33 discriminates between RALB functions in autophagy and innate immune response. Nat Cell Biol 2013; $15: 1220-30$

4. Li B, Dewey CN. RSEM: accurate transcript quantification from RNA-Seq data with or without a reference genome. BMC bioinformatics 2011;12:323

5. Clark VE, Harmanc1 AS, Bai H, Youngblood MW, Lee TI, Baranoski JF, et al. Recurrent somatic mutations in POLR2A define a distinct subset of meningiomas. Nat Genet 2016;48:1253-9

6. Li H, Durbin R. Fast and accurate short read alignment with Burrows-Wheeler transform. Bioinformatics 2009;25:1754-60

7. Li H, Handsaker B, Wysoker A, Fennell T, Ruan J, Homer N, et al. The Sequence Alignment/Map format and SAMtools. Bioinformatics 2009;25:2078-9

8. Koboldt DC, Zhang Q, Larson DE, Shen D, McLellan MD, Lin L, et al. VarScan 2: somatic mutation and copy number alteration discovery in cancer by exome sequencing. Genome Res 2012;22:568-76

9. Cingolani P, Platts A, Wang $1 \mathrm{~L}$, Coon M, Nguyen T, Wang L, et al. A program for annotating and predicting the effects of single nucleotide polymorphisms, SnpEff: SNPs in the genome of Drosophila melanogaster strain w1118; iso-2; iso-3. Fly (Austin) 2012;6:80-92 

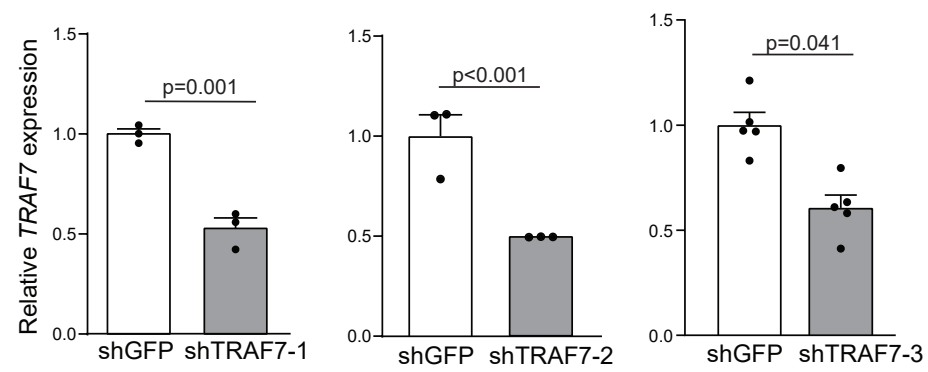

B
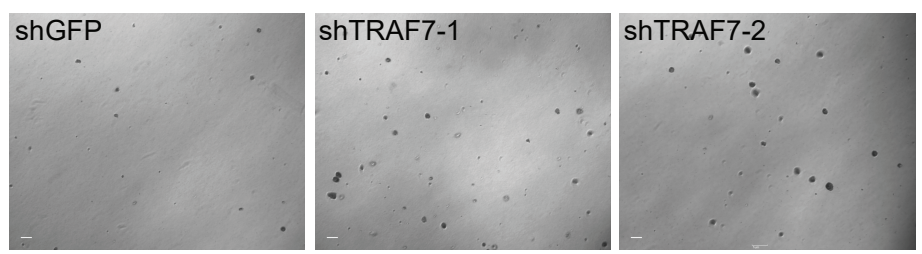

C

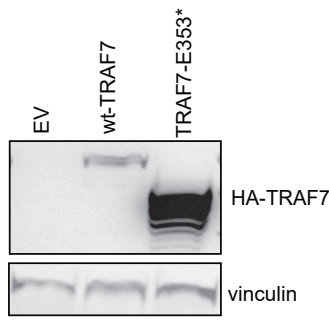

D
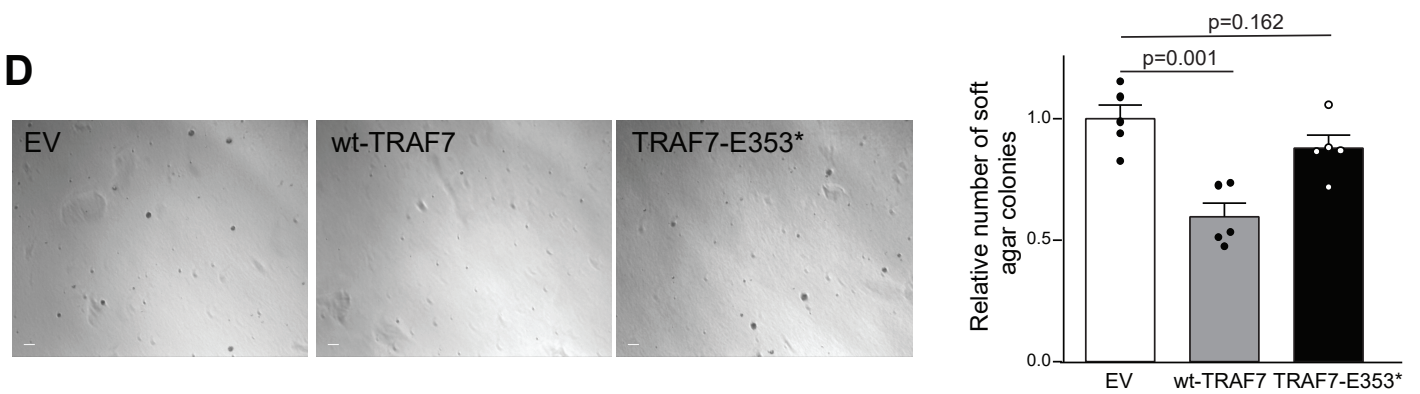

E
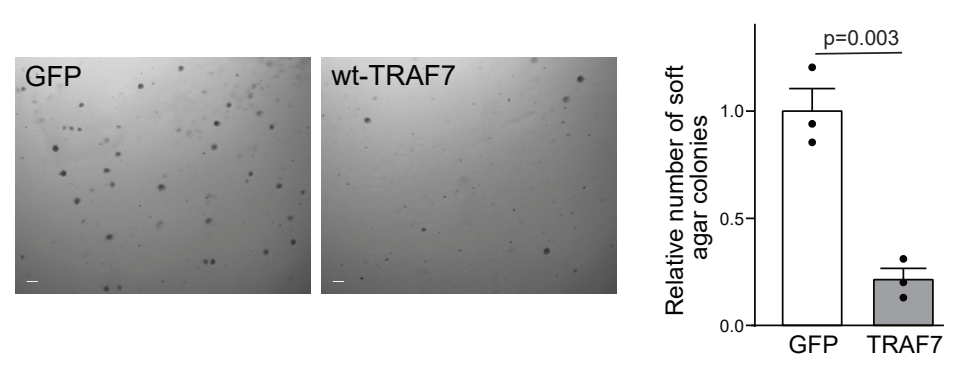

$\mathbf{F}$

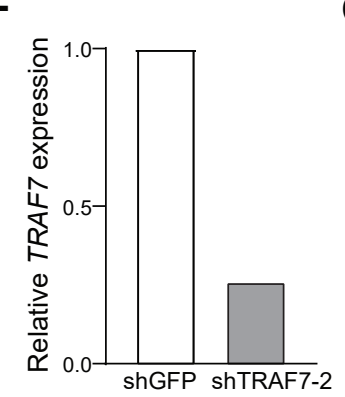

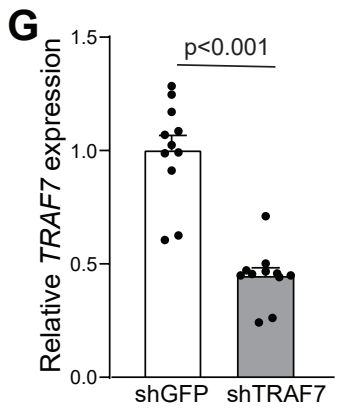

Figure S1. TRAF7 functions as a tumor suppressor in meningeal cells. A, RT-qPCR analysis of TRAF7 expression in HMCs expressing shRNA targeting GFP or TRAF7. Values are means $\pm \mathrm{SEM} ; n \geq 3$. $P$ value is calculated by a two-sided unpaired Student's $t$ test. B, Images of Al colonies of HMCs expressing shRNA against GFP or TRAF7 in soft agar. Scale bar, $100 \mu \mathrm{m}$. C, Immunoblotting analysis of HMCs expressing an empty vector (EV), wt-TRAF7, or TRAF7-E353* mutants. D, Images of Al colonies of HMCs expressing EV, wt-TRAF7, or TRAF7-E353* in soft agar. Scale bar, $100 \mu \mathrm{m}$. Relative Al growth is shown as means $\pm \mathrm{SEM} ; \mathrm{n} \geq$ $3 \mathrm{P}$ values are calculated by a two-sided Student's $\mathrm{t}$ test. E, Images of Al growth of $\mathrm{CH} 157-\mathrm{MN}$ cells expressing GFP or wt-TRAF7 in soft agar. Scale bar, $100 \mu \mathrm{m}$. Relative Al growth is shown as means \pm SEM; $n=3$. $P$ values are calculated by a two-sided Student' t test. F, RT-qPCR analysis of TRAF7 expression of CH157-MN cells expressing shGFP or shTRAF7-2. $\mathrm{n}=1$. G, RT-qPCR analysis of TRAF7 expression of HMCs expressing shGFP or shTRAF7-1 and shTRAF7-2. Values are means \pm SEM, $\mathrm{n}>10$. $\mathrm{P}$ values are from a two-sided unpaired Student's t test. 

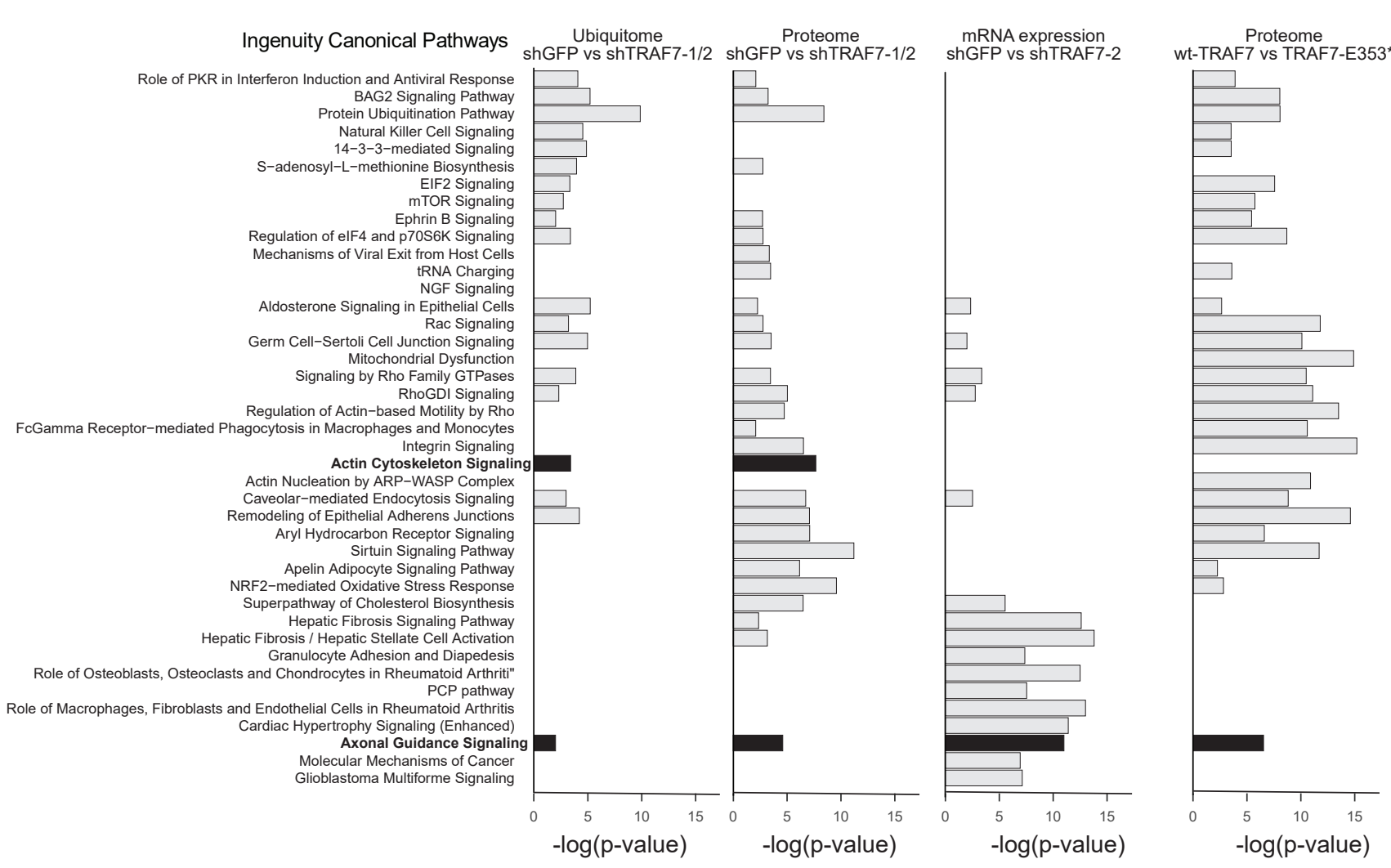
Mitochondrial Dysfunction crophages and Monocytes Actin Nucleati Caveolar-mediated Endocytosis Signaling

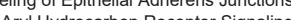
Aryl Hydrocarbon Receptor Signaling Superpathway of Cholesterol Biosynthesis Endothelial Cells in Rheumatoid Arthritis Axonal Guidance Signaling -log(p-value) -log(p-value) -log(p-value)

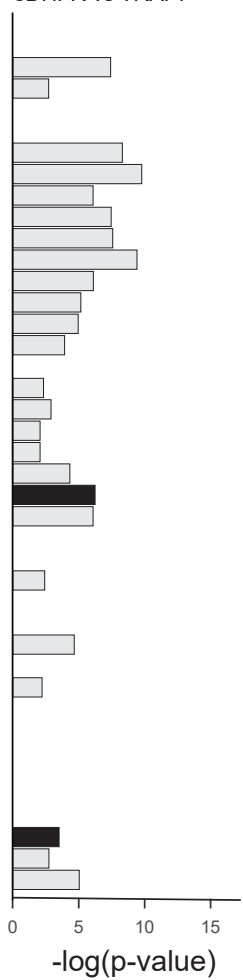

Figure S2. Canonical IPA pathways modulated by TRAF7. Ingenuity Pathway Analysis (IPA) of TRAF7-mediated alterations and TRAF7 interactome. Top 10 significantly enriched pathways for each of the datasets are shown. $P$ value $<0.01$ was used to determine the significance. The - $\log 10$ (P value) was not shown if the pathway was not significantly enriched. 
A

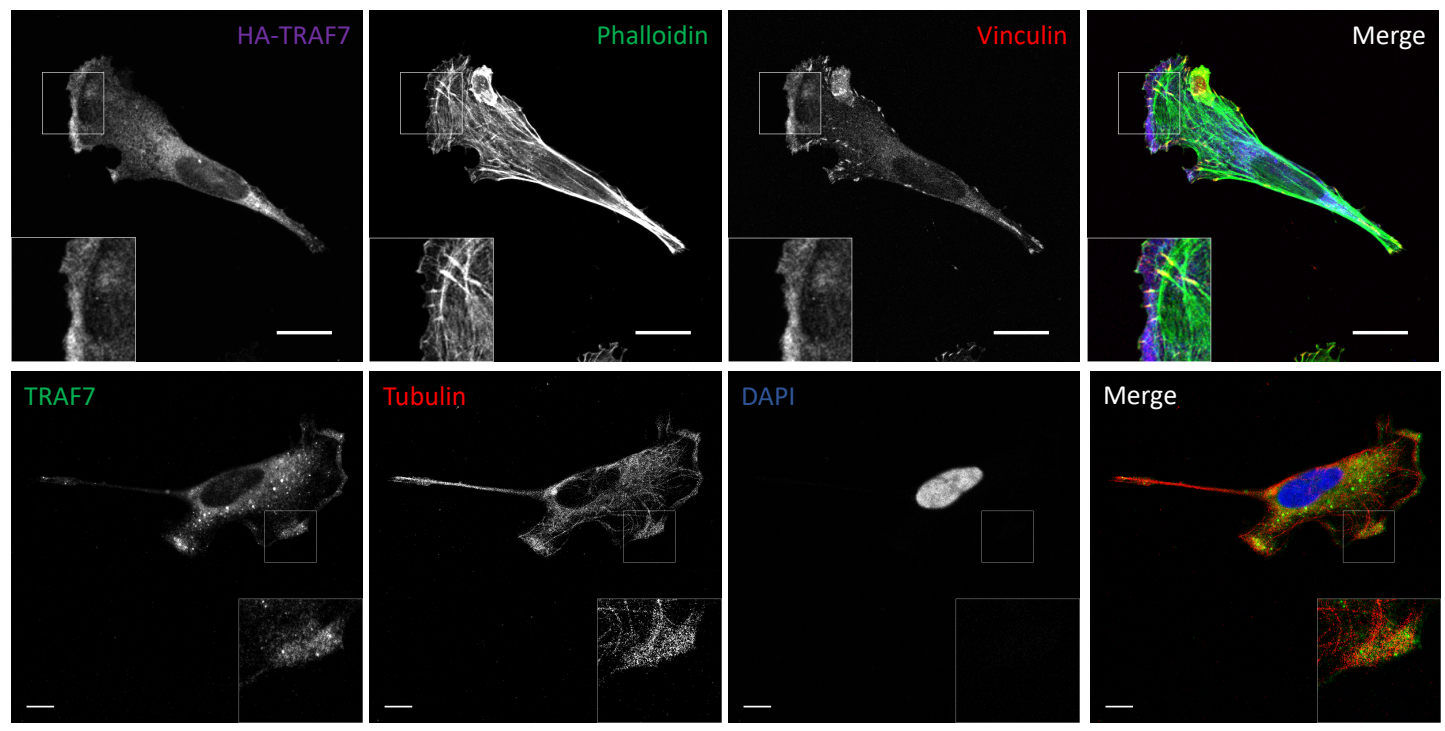

B
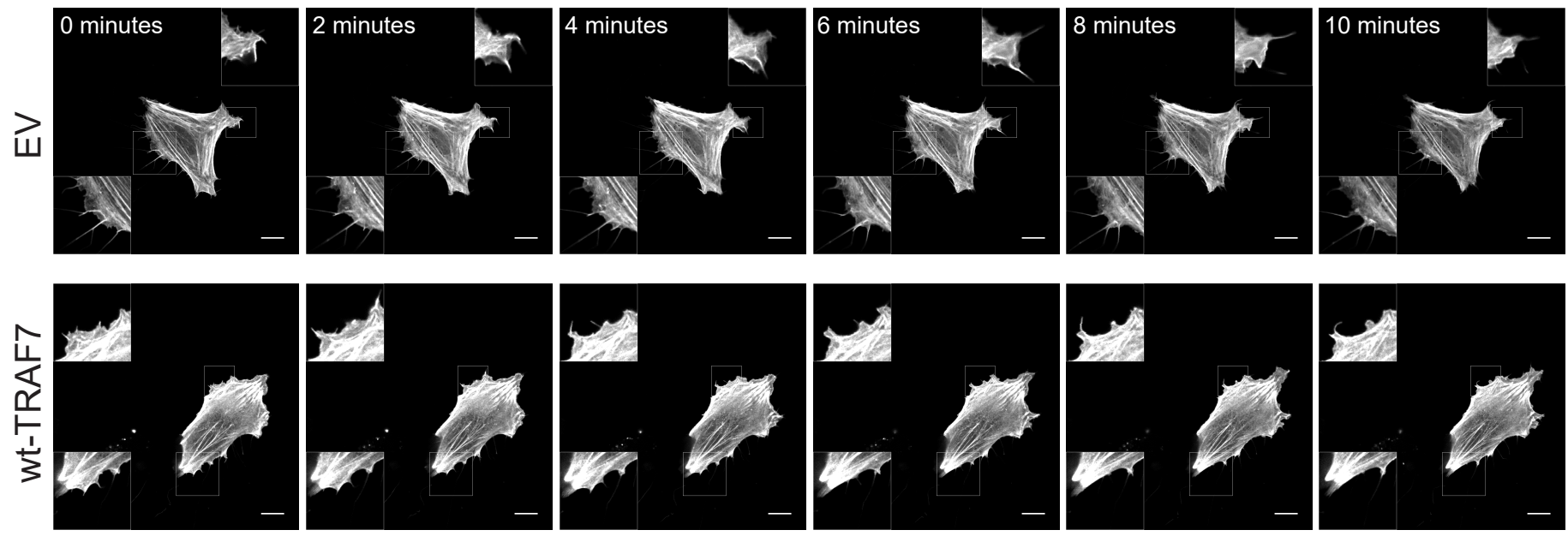

Figure S3. TRAF7-mediated alterations of actin dynamics. A, Immunostaining of HMC expressing HA-TRAF7 with anti-HA, anti-vinculin, or anti-tubulin antibodies, and phalloidin. Scale bar, 10 $\mu \mathrm{m}$. B, Human meningeal cells expressing an empty vector (EV) or TRAF7 were transiently transfected with tdTomato-Lifeact-7 and imaged in real time by confocal microscopy for over $10 \mathrm{~min}$. Scale bar, $10 \mu \mathrm{m}$. 
A

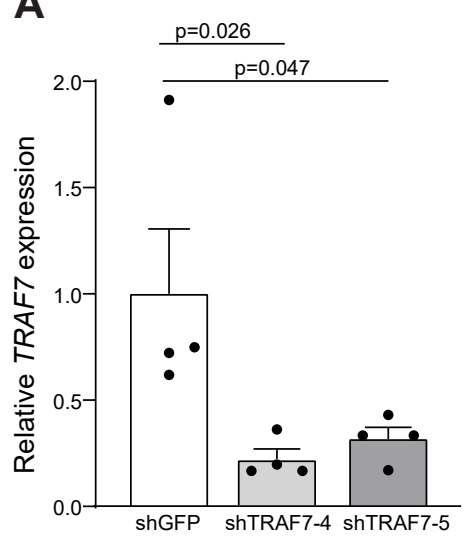

B

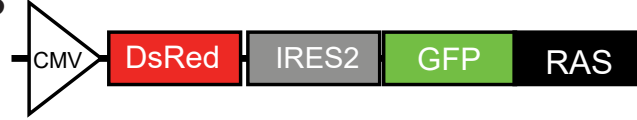

C

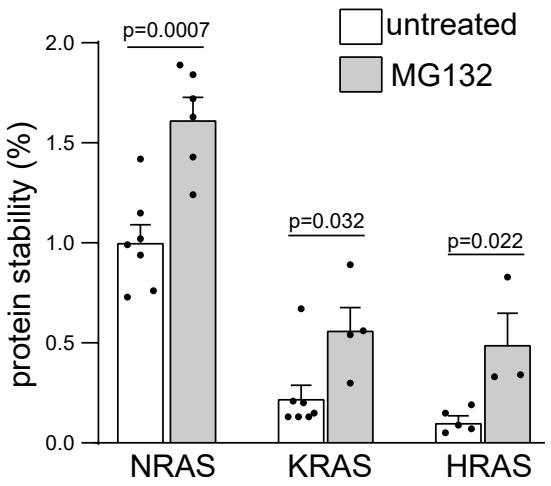

D

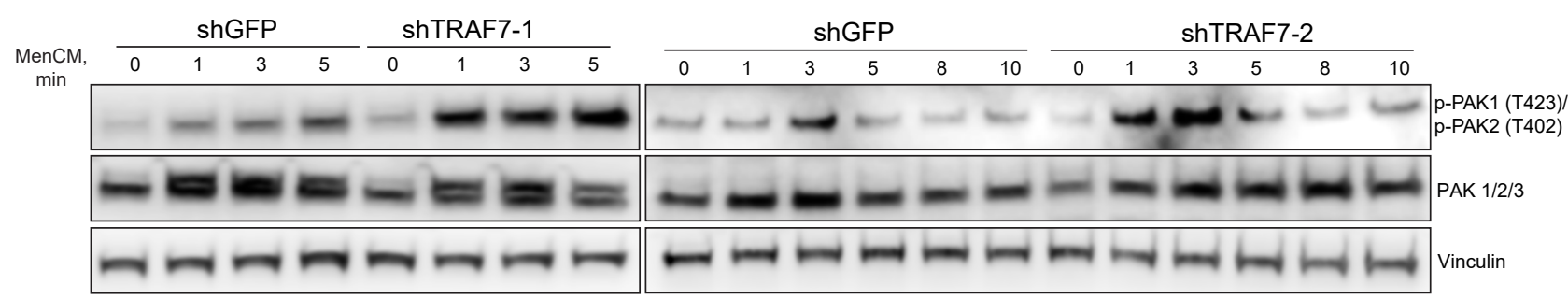

E

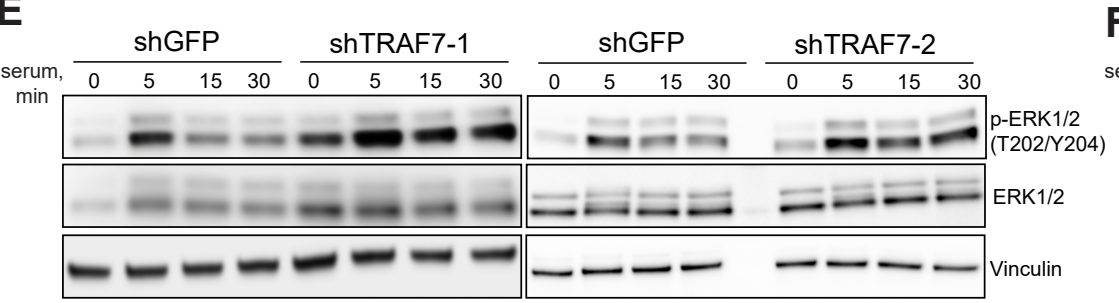

$\mathbf{F}$

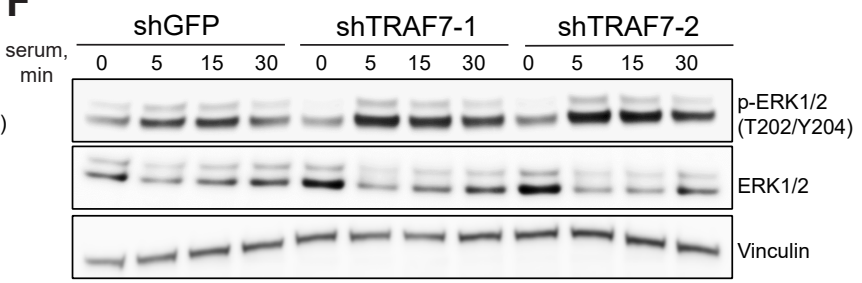

G

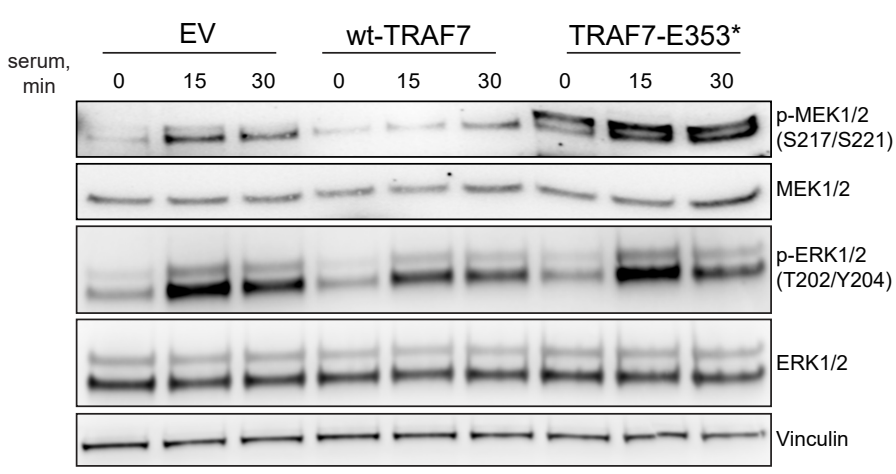

H

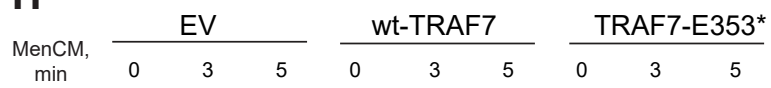

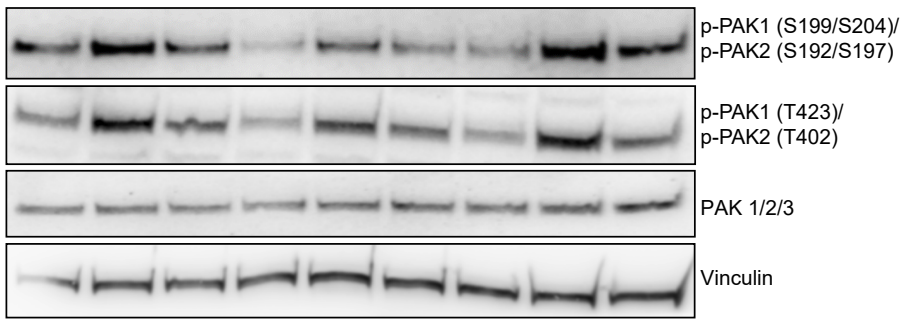

Figure S4. TRAF7 regulates proteostasis and activity of the RAS-like GTPases. A, RT-qPCR analysis of TRAF7 expression in HEK293T cells expressing shRNAs targeting GFP or TRAF7. Values are means $\pm \mathrm{SEM} ; \mathrm{n}=4$. $\mathrm{P}$ values are from one-way ANOVA. B, Schematic representation of the stability reporter system containing DsRed, followed by the IRES sequence and GFP-fused RAS isoforms or CDC42. C, Global protein stability (GPS) analysis of relative levels of RAS proteins in HEK293T cells in the absence or presence of MG132 (48 hours). Values are means $\pm S E M ; n \geq 3$. $P$ values are from a two-sided unpaired Student's t test. D, Serum-starved HMCs expressing shRNA against GFP or TRAF7 were stimulated with MenCM for the indicated time periods. Immunoblotting analysis using the indicated antibodies. E, Serum-starved HMCs expressing shRNA against GFP or TRAF7 were stimulated with $2 \%$ serum for the indicated time periods. Immunoblotting analysis using the indicated antibodies. F, CH157-MN cells expressing shGFP or shTRAF7 were serum-starved overnight, and then stimulated with $10 \%$ serum for the indicated time periods. Immunoblotting analysis was performed using the indicated antibodies. G, HMCs expressing an empty vector (EV), wt-TRAF7, or TRAF7-E353* mutant were serum-starved overnight, and then stimulated with $2 \%$ serum for the indicated time periods. Immunoblotting analysis was performed using the indicated antibodies. H, HMCs expressing EV, wt-TRAF7, or TRAF7-E353* mutant were serum-starved overnight, and then stimulated with MenCM, (Meningeal Cell Medium) for the indicated time periods. Immunoblotting analysis was performed using the indicated antibodies. 
A

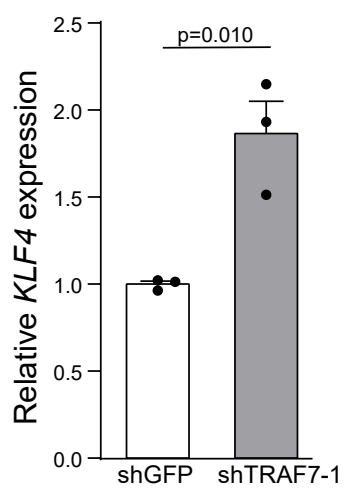

B

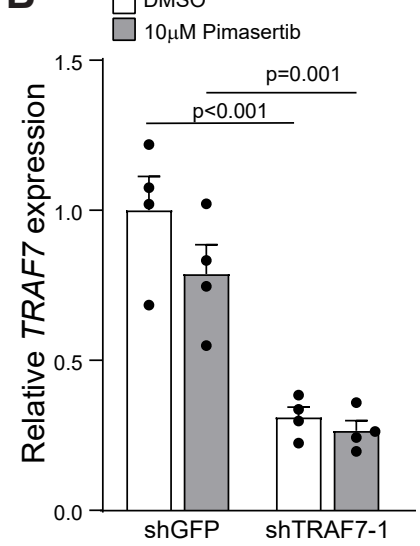

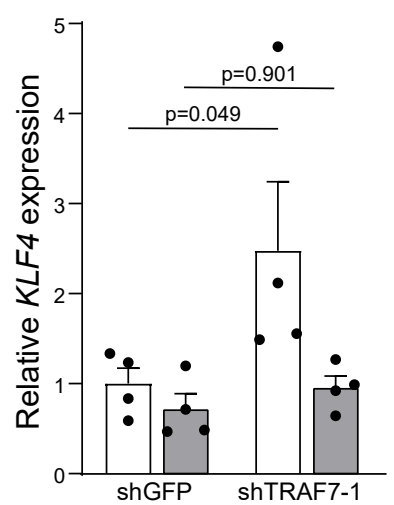

D
C

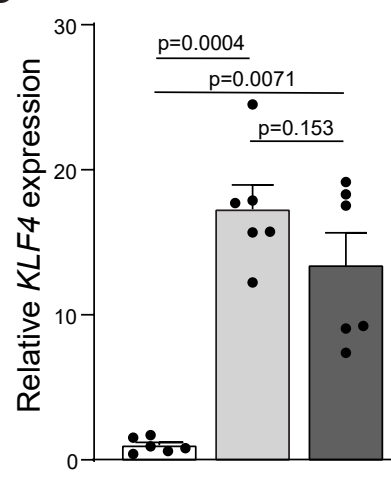

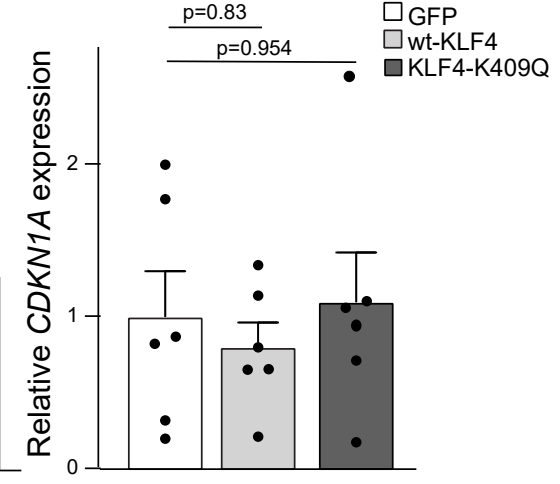

$\mathbf{E}$

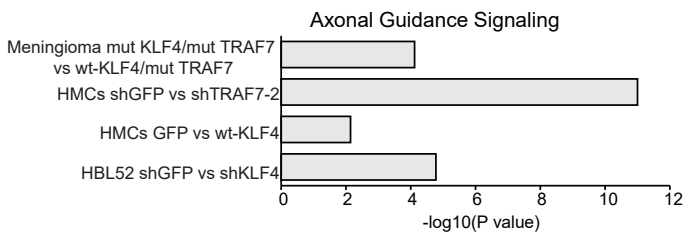

Figure S5. TRAF7 loss of function promotes KLF4 expression. A, RT-qPCR analysis of KLF4 expression in human meningeal cells expressing shGFP or shTRAF7-1. Values are mean \pm SEM. $\mathrm{n}=3$. $\mathrm{P}$ values are from two-sided unpaired Student's t test. B, RT-qPCR analysis of KLF4 and TRAF7 expression in $\mathrm{CH} 157-\mathrm{MN}$ cells expressing shGFP or shTRAF7-1 treated with DMSO or Pimasertib (10 $\mu \mathrm{M}, 48$ hours). $\mathrm{n}=4$. $\mathrm{P}$ values are from two-way ANOVA. C, RT-qPCR analysis of KLF4 and CDKN1A expression in human meningeal cells expressing GFP, wt-KLF4, or KLF4-K409Q. Values are mean \pm SEM. $\mathrm{n}=6$. $\mathrm{P}$ values are from one-way ANOVA. $\mathrm{D}, \mathrm{RT}$-qPCR analysis of $K L F 4$ expression in HBL-52 cells expressing shRNA targeting GFP or KLF4. Values are mean \pm SEM. $\mathrm{n}=4$. $\mathrm{P}$ values are from two-sided unpaired Student's $t$ test. E, The Axonal Guidance Pathway alterations in the indicated datasets. 
A

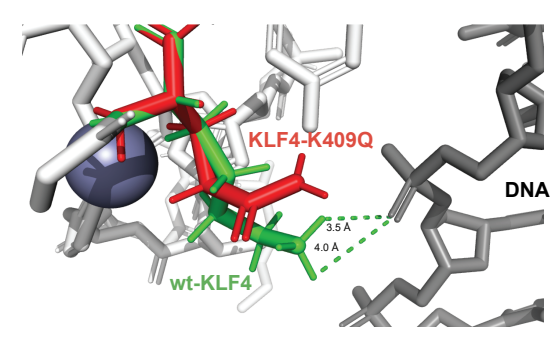

B

\begin{tabular}{|l|l|c|c|}
\hline Sequence Aligment & gRNA sequence targeting KLF4 & Indel \% & $\begin{array}{c}\text { Knock-out } \\
\text { score }\end{array}$ \\
\hline gSCR-1 vs gKLF4-1 & GTGGTGGCGCCCTACAACGG & $88 \%$ & 78 \\
\hline gSCR-2 vs gKLF4-1 & GTGGTGGCGCCCTACAACGG & $87 \%$ & 77 \\
\hline gSCR-3 vs gKLF4-1 & GTGGTGGCGCCCTACAACGG & $87 \%$ & 77 \\
\hline gSCR-1 vs gKLF4-2 & AGCCCGCGTAATCACAAGTG & $59 \%$ & 54 \\
\hline gSCR-2 vs gKLF4-2 & AGCCCGCGTAATCACAAGTG & $59 \%$ & 54 \\
\hline gSCR-3 vs gKLF4-2 & AGCCCGCGTAATCACAAGTG & $59 \%$ & 55 \\
\hline
\end{tabular}

C

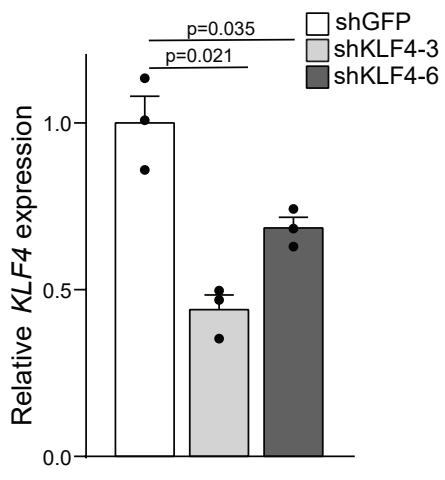

$\mathbf{E}$

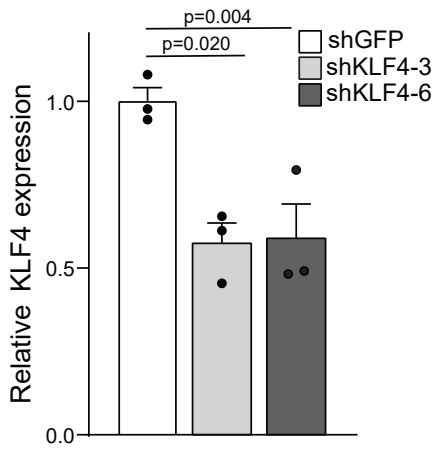

D
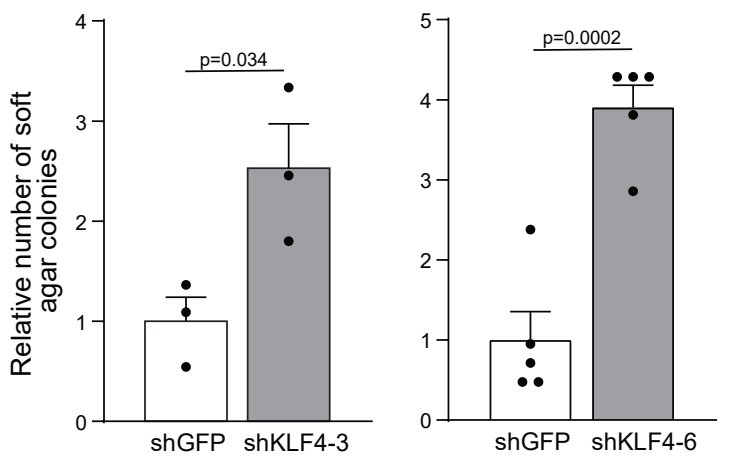

$\mathbf{F}$

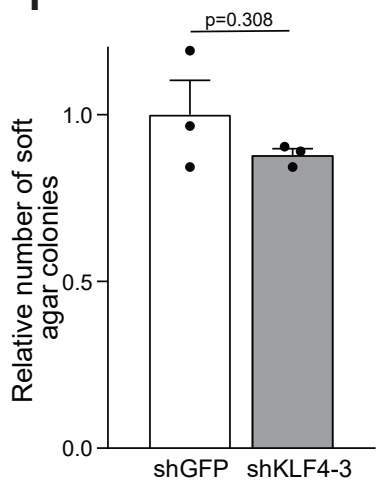

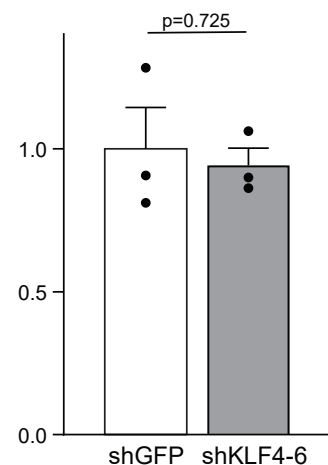

Figure S6. KLF4 promotes transformation of TRAF7 mutated cells. A, PYMOL in-silico modelling showing interaction between K409 in wt-KLF4 (PDB: 2WBU) or Q409 in KLF4-K409Q mutant and DNA. B, Indel efficiency in HMCs expressing the indicated Scramble gRNAs (gSCR) or gRNAs targeting KLF4 (gKLF4) determined the ICE v2 CRISPR Analysis Tool. C, RT-qPCR analysis of KLF4 expressions in HBL-52 cells expressing shRNA against GFP or KLF4. Values are mean \pm SEM. $n=3$. $P$ values are calculated by two-sided unpaired Student's $t$ test. D, Anchorage independent growth of HBL-52 cells expressing the indicated constructs. Values are means \pm SEM. $n=3$. $P$ values are from two-sided unpaired Student's $t$ test. E, RT-qPCR analysis of KLF4 expressions in HMCs expressing shRNA against GFP or KLF4. Values are mean $\pm S E M$. $n=3$. $P$ values are calculated by two-sided unpaired Student's $t$ test. F, Anchorage independent growth of HMCs expressing shRNA against GFP or KLF4. Values are means \pm SEM. $n=3$. $P$ values are from two-sided unpaired Student's $t$ test. 
A
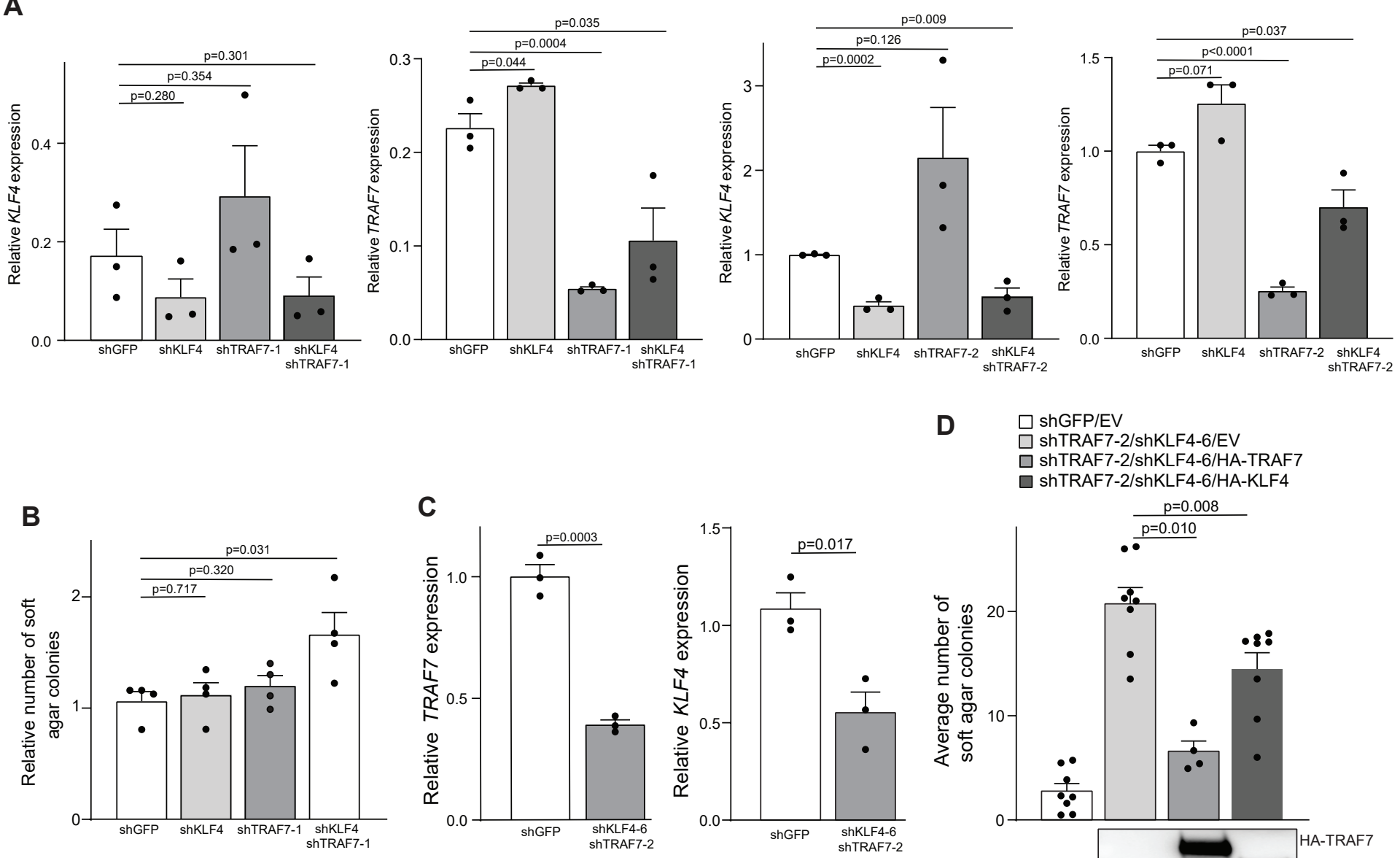

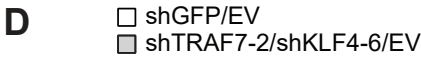
$\square$ shTRAF7-2/shKLF4-6/HA-TRAF7
$\square$ shTRAF7-2/shKLF4-6/HA-KLF4

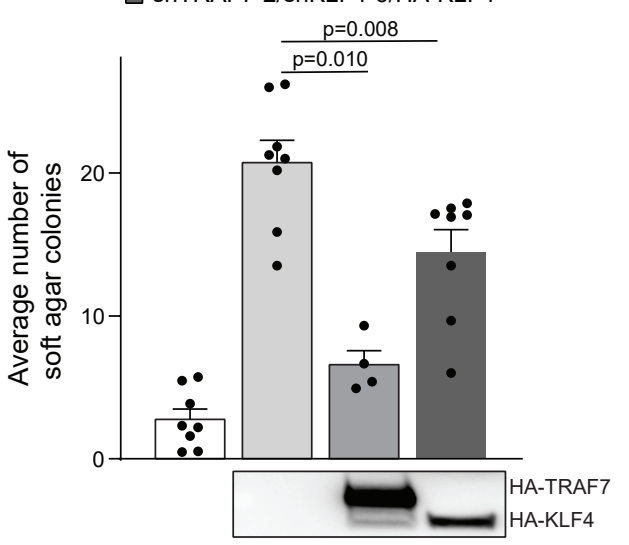

Figure S7. TRAF7 and KLF4 cooperates to promote tumorigenic transformation. A, RT-qPCR analysis of KLF4 or TRAF7 expressions in $\mathrm{CH} 157-\mathrm{MN}$ cells expressing the indicated constructs. Values are mean $\pm \mathrm{SEM} . \mathrm{n}=3$. $\mathrm{P}$ values are from two-sided unpaired Student's t test. B, Anchorage independent growth of $\mathrm{CH} 157-\mathrm{MN}$ cells expressing the indicated constructs. Values are means \pm SEM. $n=4$. $P$ values are from two-sided unpaired Student's test. C, RT-qPCR analysis of KLF4 or TRAF7 expressions in CH157-MN cells expressing the indicated constructs. Values are mean \pm SEM. $n=3$. P values are from two-sided unpaired Student's $\mathrm{t}$ test. D, Anchorage independent growth of $\mathrm{CH} 157-\mathrm{MN}$ cells expressing the indicated constructs. Values are means $\pm S E M$. $n \geq 4$. $P$ values were detected by two-way ANOVA. Immunoblot analysis showed overexpression of HA-TRAF7 or HA-KLF4 using anti-HA antibodies. 